\title{
Sums over topological sectors and
}

\section{quantization of Fayet-Iliopoulos}

\section{parameters}

\author{
Simeon Hellerman ${ }^{1}$ and Eric Sharpe ${ }^{2}$ \\ ${ }^{1}$ Institute for Physics and Mathematics of the Universe (IPMU), 5-1-5 \\ Kashiwanoha, Kashiwa 277-8583, Japan \\ simeon.hellerman.1@gmail.com \\ ${ }^{2}$ Physics Department, Virginia Tech, Blacksburg, VA 24061, USA \\ ersharpe@vt.edu
}

\begin{abstract}
In this paper we discuss quantization of the Fayet-Iliopoulos parameter in supergravity theories with altered nonperturbative sectors, which were recently used to argue a fractional quantization condition. Nonlinear sigma models with altered nonperturbative sectors are the same as nonlinear sigma models on special stacks known as gerbes. After reviewing the existing results on such theories in two dimensions, we discuss examples of gerby moduli "spaces" appearing in four-dimensional field theory and string compactifications, and the effect of various dualities. We discuss global topological defects arising when a field or string theory moduli space has a gerbe structure. We also outline how to generalize the results of Bagger-Witten and more recent authors on quantization issues in supergravities from smooth moduli spaces to smooth moduli stacks, focusing particular attention on stacks that have gerbe structures.
\end{abstract}

e-print archive: http://lanl.arXiv.org/abs/1012.5999v2 


\section{Contents}

1 Introduction

2 Review of two-dimensional theories with altered topological sectors

3 Four-dimensional physics

4 Examples, duality in gerby moduli "spaces"

4.1 Field theory

4.2 String theory

5 Topological defects and gerby moduli spaces

1164

6 Consistency conditions on classical supergravity

1167

6.1 Review of standard supergravity case

1167

6.2 Generalization to smooth Deligne-Mumford stacks

7 Conclusions

Acknowledgments

1179

Appendix A. Four-dimensional decomposition conjecture

Appendix B. Two-dimensional BF theory and cluster decomposition

B.1 Hilbert space of the BF theory

B.2 Path integral measure on a finite torus

1182

B.3 Path integral over nonzero modes

B.4 Integral over the zero modes

B.5 Hilbert space interpretation of the vacuum amplitude 


\section{B.6 Spectrum and commutation relations of local operators and line operators}

\section{B.7 Commutation relations}

\section{B.8 Direct demonstration of non-cluster- decomposition}

\section{References}

\section{Introduction}

Recently, there has been much progress in understanding Fayet-Iliopoulos parameters in supergravity, generalizing work of, e.g., Bagger-Witten [1], see for example [2-9]. In particular, the recent paper [7] argued that in the special case of linearly realized group actions, Fayet-Iliopoulos parameters could be interpreted as charges for a $U(1)$ gauge symmetry, and so are quantized. This result was generalized in [8] to the more nearly generic case of nonlinearly realized group actions, by demonstrating that the FayetIliopoulos parameters determine the lift of the group action to the BaggerWitten [1] line bundle. As such lifts of group actions are quantized, the Fayet-Iliopoulos parameters are therefore also quantized.

This paper will focus on another aspect of [7], specifically a proposal for Fayet-Iliopoulos quantization when the moduli space is defined by twodimensional sigma models with a restriction on allowed instantons. Such two-dimensional theories have been discussed previously in, e.g., [10-16], and are the same as sigma models on gerbes, special kinds of stacks.

Schematically, smooth stacks are "manifolds paired with automorphisms." Stacks admit metrics, spinors, and all the other structures appearing in classical field theories. The original interest in stacks in the physics community revolved around using them to form new string compactifications, new conformal field theories, and applying them to give a more fundamental understanding of certain existing compactifications.

Previous work on consistency conditions in supergravity theories has assumed that the moduli space is a smooth manifold. However, in mathematics, moduli "spaces" are usually stacks, and not manifolds, so to have a broad understanding of classical consistency conditions on supergravity theories, one must understand cases in which the moduli "space" of the supergravity is a stack. This paper is a step in a program of understanding consistency conditions for such more general cases. 
To be more specific, in this paper we will discuss generalizations of consistency conditions on supergravities from moduli spaces that are manifolds to moduli "spaces" that are smooth Deligne-Mumford stacks, focusing particular attention on stacks that are gerbes over manifolds. That said, in typical examples arising in string compactifications, the moduli stack has singularities, so our generalization to stacks will still not describe all cases pertinent to string compactifications, but is a step towards a directly pertinent treatment.

We begin in Section 2 by reviewing two-dimensional sigma models on stacks, focusing in particular on gerbes over manifolds. Two-dimensional sigma models on gerbes over manifolds look like sigma models on the underlying manifolds but with a restriction on topological sectors. These have been discussed in considerable detail in both the mathematics and physics literature, as we review.

In Section 3 we discuss analogous four-dimensional theories. There are some significant differences between two-dimensional and four-dimensional cases, including issues around presentation dependence, and (on $\mathbf{R}^{4}$ ) a lack of nonperturbative sectors in gerbe theories.

In Section 4 we discuss particular examples of both field and string theories whose moduli "spaces" are gerbes over manifolds. In particular, previous work on gerbe structures in supergravity moduli spaces [7] did not give any examples of string compactifications in which such structures would arise, which we remedy here. We discuss the physical impact of such gerbe structures, and also discuss the action of duality groups.

In Section 5 we discuss global topological defects in theories with gerby moduli spaces. Topological defects are classified by homotopy of the moduli space, and gerbe structures contribute nontrivially to the homotopy. We discuss whether the contributions to homotopy from gerbe structures have physical meaning.

In Section 6 we outline how to generalize consistency conditions on classical supergravities in $[1,8]$ to moduli "spaces" that are smooth DeligneMumford stacks, focusing in particular on the case of stacks that are gerbes. In particular, we discuss the case of Bagger-Witten [1] line bundles that are "fractional" over the gerby moduli space.

In Appendix A we discuss a four-dimensional analogue of the "decomposition conjecture" [14] that plays a vital role in understanding two-dimensional sigma models on gerbes. In this four-dimensional analogue, we restrict sums over four-dimensional instantons - as a result, the four-dimensional version 
is not directly relevant to four-dimensional sigma models on gerbes, but nevertheless we thought it appropriate to discuss here.

Finally, in Appendix B we discuss two-dimensional BF theory and analogues of gerbe structures and decomposition statements there. This gives us an opportunity to discuss the relationship between locality and cluster decomposition in an explicit example.

While this work was being completed, the paper [9] appeared, which has nontrivial overlap.

\section{Review of two-dimensional theories with altered topological sectors}

The recent paper [7] discussed theories defined by restricting sums over instantons to a subset of all instantons. In this section we briefly review some of the previous work done on such theories.

In the case of two-dimensional nonlinear sigma models, a nonlinear sigma model in which the sum over worldsheet instantons is restricted to a subset of all instantons is the same as a string on a gerbe, a special kind of stack, as is discussed in the physics literature in for example [10-18] and reviewed in conference proceedings including [19-21]. (There is also a significant mathematics literature on Gromov-Witten invariants of stacks and gerbes; see for example [22-25] for a few representative examples.)

Briefly, a stack is a manifold "paired with automorphisms." (See, e.g., [26-28] for a more technical definition.) At the same level of brevity, a gerbe is a stack in which one has the same automorphisms everywhere. Mathematically, a gerbe can be thought of locally as covered by patches of the form $[U / G]$ where $U$ is an open set and $G$ acts trivially on $U$. Stacks keep track of even trivial group actions, and so $[U / G]$ is distinguished (as a stack) from just $U$.

One of their properties that plays a role in this paper is that if $\mathcal{G}$ is a gerbe over a manifold $M$, then maps into $\mathcal{G}$ are equivalent to maps into $M$ with a restriction on their degree, as discussed in for example [13]. Briefly, a map from any space $X$ into a gerbe $\mathcal{G}$ over $M$ is equivalent ${ }^{1}$ to a map

\footnotetext{
${ }^{1}$ There is a closely related statement for bundles. Given a map $g: X \rightarrow E$ for some bundle $\pi: E \rightarrow M$, we can compose with $\pi$ to produce a map $f: X \rightarrow M$. Furthermore, we can define a trivialization of $f^{*} E$ canonically: recall

$$
f^{*} E \equiv\{(x, e) \in X \times E \mid f(x)=\pi(e)\}
$$
}


$f: X \rightarrow M$ into the underlying space, together with a trivialization of $f^{*} \mathcal{G}$. For a $\mathbf{Z}_{k}$ gerbe, say, $f$ induces a map

$$
f^{*}: H^{2}\left(M, \mathbf{Z}_{k}\right) \longrightarrow H^{2}\left(X, \mathbf{Z}_{k}\right)
$$

which maps the characteristic class of the gerbe $\mathcal{G}$ (an element ${ }^{2}$ in $H^{2}(M$, $\left.\mathbf{Z}_{k}\right)$ ) to the characteristic class of $f^{*} \mathcal{G}$, which should vanish (otherwise $f^{*} \mathcal{G}$ would not admit a trivialization). For example, if $X=\mathbf{P}^{1}$ and $M=\mathbf{P}^{N-1}$, then $f$ is characterized by an integer, its degree. In this case, $f^{*}$ acts by multiplication by the degree of $f$, so if we let $n$ denote the characteristic class of the gerbe $(\bmod k)$, and $d$ the degree of $f$, then we have the constraint

$$
d(n \bmod k)=0 \bmod k .
$$

In other words, $d n$ must be a multiple of $k$, a constraint on the allowed degrees of maps $f$. (Note the constraint depends upon the characteristic class of the gerbe - for example, for a trivial gerbe, $n \equiv 0 \bmod k$, and so there is no constraint.)

In mathematics, moduli "spaces" are usually stacks, hence one should not be surprised to find stack structures arising in moduli spaces of interest to physicists. Indeed, in this paper we shall discuss examples of moduli "spaces" with nontrivial stack structures arising in physics, and their analysis.

In previous work, e.g., [11-13], two-dimensional sigma models on smooth Deligne-Mumford stacks were defined physically by using the fact that essentially $^{3}$ all such stacks can be described as quotients $[X / G]$ for $X$ some space and $G$ some group acting on $X$. The special case of a gerbe corresponds to a subgroup of $G$ acting trivially on $X$. (We shall review how physics keeps track of even trivial group actions.) To such a quotient we associate a

so we can define a trivialization $X \rightarrow f^{*} E$ by $x \mapsto(x, g(x))$ for $x \in X$. Conversely, if $E$ is a bundle over a space $M$, and we are given a map $f: X \rightarrow M$ and a trivialization of $f^{*} E$, then since there is a canonical map $f^{*} E \rightarrow E$, the trivialization $X \rightarrow f^{*} E$ can be composed with that canonical map to form a map $g: X \rightarrow E$, whose composition with the projection is $f$ again.

${ }^{2}$ In general, the characteristic class of a $G$-gerbe on a manifold $X$ is an element of $H^{2}\left(X, C^{\infty}(G)\right)$. Here, since $G$ is assumed finite, $C^{\infty}(G)=G$. For $U(1)$ gerbes,

$$
H^{2}\left(X, C^{\infty}(U(1))\right)=H^{3}(X, \mathbf{Z})
$$

and in this fashion one recovers the usual physics description in terms of the curvature $H$ of the $B$ field.

${ }^{3}$ See [12] for a discussion of rare counterexamples, and their apparent lack of physical relevance. 
$G$-gauged sigma model on $X$. A given stack can admit multiple presentations of this form; we associate universality classes of renormalization group flow to particular stacks. Much effort was expended in previous work to check presentation-independence of universality classes.

A standard example ${ }^{4}$ of a gerbe is a $\mathbf{Z}_{k}$ gerbe on a projective space, defined by a supersymmetric $\mathbf{P}^{n-1}$ model, a linear gauge theory, with $n$ chiral superfields $\Phi_{i}$ each of charge $k$ instead of charge 1 . As discussed in [11], in two dimensions such theories are nonperturbatively distinct from the ordinary $\mathbf{P}^{n-1}$ model. On a noncompact worldsheet, this can be seen by thinking about periodicity of the two-dimensional theta angle - such theories can be distinguished by the existence of massive minimally charged objects, which alter the periodicity.

Let us work through this argument in more detail. As described in [11, Section 3], since in two dimensions the $\theta$ angle couples to $\operatorname{Tr} F$, we can determine the instanton numbers through the periodicity of $\theta$. Suppose we have the physical theory described above, namely a GLSM with Higgs fields of charge $k$, plus two more massive fields, of charges +1 and -1 . In a two-dimensional theory, the $\theta$ angle acts as an electric field, which can be screened by pair production, and that screening determines the periodicity of $\theta$. If the only objects we could pair produce were the Higgs fields of charge $k$, then the theta angle would have periodicity $2 \pi k$, and so the instanton numbers would be multiples of $1 / k$. However, since the space is noncompact, and the electric field fills the entire space, we can also pair produce arbitrary numbers of the massive fields, which have charges \pm 1 , and so the $\theta$ angle has periodicity $2 \pi$, so the instantons have integral charges. In particular, even if the masses of the massive fields are beyond the cutoff scale, the theta angle periodicity can still know about them, and so they can still help determine the low-energy effective field theory.

We can phrase this more simply as follows. In a theory with only Higgs fields of charge $k$, the instanton numbers are multiples of $1 / k$, and so the resulting physics is equivalent to that of a GLSM with minimal charges. However, if we add other fields of charge \pm 1 , then the instanton numbers are integral, and if those fields become massive, and we work at an energy scale below that of the masses of the fields, then we have a theory with Higgs fields of charge $k$, and integral instanton numbers, giving us the physics that corresponds to a gerbe target. (This argument was, to our knowledge, first

\footnotetext{
${ }^{4}$ This $\mathbf{Z}_{k}$ gerbe has characteristic class $-1 \bmod k$, so from the previous analysis, maps into the gerbe are maps into the underlying projective space of degree divisible by $k$, as should also be clear from the description of the gauge theory.
} 
developed by J. Distler and R. Plesser at an Aspen summer meeting in 2004, used with their permission in [11, Section 3] and then also described much more recently in [7].)

On a compact worldsheet, this distinction between minimal and nonminimal charges is a consequence of how matter couplings are defined globally (i.e., as sections of bundles). In detail, to uniquely define the theory nonperturatively on a compact space, we must specify, by hand, the bundles that the Higgs fields couple to. If the gauge field is described by a line bundle $L$, then coupling all of the Higgs fields to $L^{\otimes k}$ is a different prescription from coupling all of the Higgs fields to $L$. As a result, the spectrum of zero modes differs between the two theories, hence correlation functions and anomalies differ between the two theories.

Some of the structure above - such as the theta angle argument - is specific to two dimensions, but some will generalize. Later, we will argue that gerbes are relevant to four-dimensional physics when either the fourdimensional spacetime is topologically nontrivial, or there are massive states which are not invariant, mirroring aspects of the two-dimensional story above.

In any event, here are a few physical consequences of this distinction between minimally and nonminimally charged theories in this two-dimensional example [13]:

- The axial $U(1)_{A}$ of the supersymmetric $\mathbf{P}^{n-1}$ model is broken to $\mathbf{Z}_{2 k n}$ by instantons instead of $\mathbf{Z}_{2 n}$, when the chiral superfields have charge $k$.

- The quantum cohomology ring is $\mathbf{C}[x] /\left(x^{k n}-q\right)$ instead of $\mathbf{C}[x] /\left(x^{n}-\right.$ $q)$, reflecting the fact that $\mathrm{A}$ model correlation functions differ. (The paper [13] provided GLSM-based physical computations of these quantities, as well as a mirror symmetry computation verification, and in addition there are also mathematical definitions and matching results; see for some representative examples [22-25].)

- The (Toda) Landau-Ginzburg mirror can be described by a superpotential with a field $\Upsilon$ valued in $k$ th roots of unity,

$$
W=e^{Y_{1}}+e^{Y_{n-1}}+\Upsilon e^{-Y_{1}-\cdots-Y_{n-1}}
$$

reflecting both the fact that the theory on a gerbe is equivalent to a theory on a disjoint union of spaces, and the fact that mirror symmetry dualizes nonperturbative effects into perturbative ones. (This result was physically derived in [13] from duality for GLSM's ala [29,30], and 
also independently derived in, e.g., [25] from mathematical considerations.)

So far we have outlined how noneffective continuous group actions can lead to new physics; the same is true of finite group actions. For example, consider the orbifold $\left[T^{6} / D_{4}\right]$ where $D_{4}$ is an eight-element group that projects onto $\mathbf{Z}_{2} \times \mathbf{Z}_{2}$ :

$$
1 \longrightarrow \mathbf{Z}_{2} \longrightarrow D_{4} \longrightarrow \mathbf{Z}_{2} \times \mathbf{Z}_{2} \longrightarrow 1 .
$$

To specify the orbifold, we must specify the action of $D_{4}$ on $T^{6}$. Let us take the $\mathbf{Z}_{2}$ center to act trivially, so that the $D_{4}$ acts by first projecting to $\mathbf{Z}_{2} \times \mathbf{Z}_{2}$, and then act with a standard Calabi-Yau action of $\mathbf{Z}_{2} \times \mathbf{Z}_{2}$ on $T^{6}$, as described in, e.g., [31]. Since the $\mathbf{Z}_{2}$ center acts trivially, one might naively assume that the $\left[T^{6} / D_{4}\right]$ orbifold would be physically equivalent to a $\left[T^{6} / \mathbf{Z}_{2} \times \mathbf{Z}_{2}\right]$ orbifold. Instead, one computes that at one-loop, for example, [14, Section 5.2]

$$
Z\left(\left[T^{6} / D_{4}\right]\right)=Z\left(\left[T^{6} / \mathbf{Z}_{2} \times \mathbf{Z}_{2}\right] \coprod\left[T^{6} / \mathbf{Z}_{2} \times \mathbf{Z}_{2}\right]_{\text {d.t. }}\right),
$$

where the subscript indicates the presence of discrete torsion in one of the two factors. We therefore see explicitly that, in this example, the string orbifold knows about the trivially acting $\mathbf{Z}_{2}$ subgroups. Many additional examples have been described in detail in the references. Thus, string orbifolds know about trivially acting subgroups, just as we saw in two-dimensional gauge theories.

Technically, in $(2,2)$ supersymmetric cases, these two-dimensional theories (in which a trivially acting group have been gauged) do not obey cluster decomposition. (This is an immediate consequence of Weinberg's ancient argument for theories with any restriction on instanton degrees, and can also be seen for CFT cases by, for example, computing massless spectra and observing multiple dimension zero operators.) This would be a problem were it not for the fact that they are equivalent to nonlinear sigma models on disjoint unions of ordinary spaces [14], a result described there as the "decomposition conjecture." The latter also do not obey cluster decomposition, but are obviously nevertheless under good control, in the sense that we can renormalize by local counterterms, and so forth. Thus, they are sums of theories which obey cluster decomposition, and so at least morally are "local" theories.

One of the original reasons for interest in these gerbe theories was the idea that they might define new SCFTs, new string compactifications. Because of the decomposition conjecture, that is not really the case in $(2,2)$ theories, 
as one gets sums of existing theories. In $(0,2)$ theories, on the other hand, the story seems to be somewhat more complex; an example is outlined in [32, Section 3.2], and a more complete description will appear in [16].

We can understand the decomposition conjecture schematically as follows. Consider a nonlinear sigma model on a space $X$, for simplicity with $H^{2}(X, \mathbf{Z})=\mathbf{Z}$, with a restriction on worldsheet instantons to degrees divisible by $k$. We can realize that restriction in the path integral by inserting a projection operator

$$
\frac{1}{k} \sum_{n=0}^{k-1} \exp \left(\mathrm{i} \int \phi^{*}\left(\frac{2 \pi n}{k} \omega\right)\right),
$$

where $\omega$ is the de Rham image of a generator of $H^{2}(X, \mathbf{Z})$. Inserting this operator into a partition function is equivalent to working with a sum of partition functions with rotating $B$ fields, and this is the essence of the decomposition conjecture.

One of the applications of the result above is to Gromov-Witten theory, where it has been checked and applied to simplify computations of GromovWitten invariants of gerbes, see [33-38]. Another application is to gauged linear sigma models [15], where it answers old questions about the meaning of the Landau-Ginzburg point in a GLSM for a complete intersection of quadrics, as well as corrects old lore on GLSMs.

\section{Four-dimensional physics}

Now, let us turn to four-dimensional theories, and discuss how the physics differs from two-dimensional cases.

To sharply compare with the two-dimensional cases outlined in the previous section, let us work through a toy example. Consider a $U(1)$ gauge theory in supergravity, ${ }^{5}$ defined over spacetime $\mathbf{R}^{4}$, with supergravity moduli space $\mathbf{C}^{2 n+2}$, describing $2 n+2$ complex scalars, on which the $U(1)$ acts as follows: $n$ fields of charge $k, n$ fields of charge $-k$, one field $\chi$ of charge +1 , one field $\tilde{\chi}$ of charge -1 . Let us furthermore assume that there is a superpotential $^{6} W=m \chi \tilde{\chi}$, giving a mass $m$ to the two fields of charge \pm 1 .

\footnotetext{
${ }^{5}$ The analysis presented here is due to J. Distler, and we thank him for allowing us to present it here.

${ }^{6}$ Since we are working in supergravity, the superpotential is a section of the BaggerWitten [1] line bundle, but since the supergravity moduli space is $\mathbf{C}^{2 n+2}$, the BaggerWitten line bundle is necessarily the trivial line bundle $\mathcal{O}$.
} 
The upshot of this construction is that at low energies, one has a $U(1)$ gauge theory with nonminimal charges, closely analogous to those discussed in the last section realizing sigma models on gerbes.

Now, one might worry that at at low energies, below the cutoff scale, perhaps all the states of the theory have charges that are a multiple of $k$. In such a case, the fact that the electron charges above are nonminimal would be physically irrelevant; at low energies, the theory would be physically equivalent to a theory in which all fields had charge 1 , not $k$. To settle this issue, we need to understand the correct electric charge quantization in this theory. In two dimensions, we could distinguish a theory with nonminimal charges from a theory with minimal charges via nonperturbative effects, invoking the theta angle periodicity to "see" states with masses beyond the cutoff. Here, by contrast, note that since we are describing a $U(1)$ gauge theory on $\mathbf{R}^{4}$, there are no $U(1)$ instantons. However, because this theory is coupled to gravity, we can appeal to the existence of ReissnerNordström black holes. We can use them to determine the correct electric charge quantization in the theory at places in its moduli space where the $U(1)$ is unbroken, and then appeal to continuity to understand the rest.

First, suppose that the mass $m<M_{\mathrm{Pl}}$. When the $U(1)$ is unbroken, there are electrically charged Reissner-Nordström black holes. Since $m<$ $M_{\mathrm{Pl}}$, microscopic black holes can Hawking radiate $\chi, \tilde{\chi}$, and so even if one started with a black hole of charge a multiple of $k$, it could Hawking radiate down to charge 1 . Thus, at least at points where the $U(1)$ is unbroken, the nonminimal charges of the Higgs fields are physically relevant. At more generic points on the moduli space, where the $U(1)$ is Higgsed to $\mathbf{Z}_{k}$, we need there to be excitations on which the $\mathbf{Z}_{k}$ acts nontrivially, and at least for small Higgs vev, the Reissner-Nordström black holes should ${ }^{7}$ become such excitations.

If $m>M_{\mathrm{Pl}}$, then the Hawking radiation process above cannot happen, but demanding that physics be continuous in $m$ leads us to believe that the electric charges of black holes are still multiples of 1 rather than $k$. Thus, again, the fact that the massless fields in the gauge theory have nonminimal charges, is physically relevant.

So far we have discussed a four-dimensional analogue of the two-dimensional theta-angle-periodicity argument for the relevance of nonminimal charges, using black holes rather than theta angles. In the rest of this section we

\footnotetext{
${ }^{7}$ In effect, we are appealing to a continuity argument. As a potential loophole, we should mention that it is known from work on wall-crossing that the spectrum of BPS states in a supersymmetric theory does not always behave so simply. We do not need to assume the states here are BPS, but it is possible that analogous processes may apply.
} 
shall discuss some subtleties specific to four-dimensional theories, and their relevance to four-dimensional sigma models on stacks.

First, let us examine more closely the (lack of) nonperturbative sectors in field theories on $\mathbf{R}^{4}$ not coupled to gravity. One of the significant properties of two-dimensional sigma models on gerbes was that the nonperturbative sector is altered: one sums over only some instantons, not all of them. However, in four dimensions, on $\mathbf{R}^{4}$, there is no constraint on nonperturbative sectors:

- We have already seen the example of a $U(1)$ gauge theory with nonminimal charges on $\mathbf{R}^{4}$. Instantons in such a theory would be described by principal $U(1)$ bundles on $S^{4}$ (the one-point compactification of $\mathbf{R}^{4}$, taken to encode compact-support issues), and there are no nontrivial principal $U(1)$ bundles on $S^{4}$, because $H^{2}\left(S^{4}, \mathbf{Z}\right)$ vanishes. (Moreover, on $\mathbf{R}^{4}$ or $S^{4}$, for a $U(1)$ gauge theory the quantity $\int \operatorname{Tr} F \wedge F$ vanishes identically for all gauge field configurations appearing in the path integral (i.e., compactly supported ones), not just saddle points.)

- Next, formally consider a nonlinear sigma model summing over maps $\mathbf{R}^{4} \rightarrow \mathcal{G}$ for $\mathcal{G}$ some $G$-gerbe (for finite $G$ ) over a smooth manifold $M$. Again for compact-support reasons we can replace $\mathbf{R}^{4}$ by $S^{4}$ for the purposes of mathematical computations. As discussed in the last section, a map $X \rightarrow \mathcal{G}$ for any space $X$ is the same as a map $f: X \rightarrow$ $M$ together with a trivialization of $f^{*} \mathcal{G}$, and demanding that $f^{*} \mathcal{G}$ be trivializable restricts possible maps $f$. In the present case, however, since $H^{2}\left(S^{4}, \mathbf{Z}\right)$ vanishes, the pullback $f^{*} \mathcal{G}$ is always trivializable for any $\mathcal{G}$, and so demanding that $f^{*} \mathcal{G}$ be trivial is no longer a constraint on possible maps.

In particular, unlike two dimensions, on $\mathbf{R}^{4}$ there is no reason to believe that cluster decomposition will necessarily be violated, and there is no analogue of the decomposition conjecture [14] for gerbe theories.

Next, let us consider a technical point in the renormalization-group flow of the low-energy effective gauged sigma models arising in this and analogous examples. (We would like to thank J. Distler for patient explanations of this point.) Schematically, if $v$ is the scale of the Higgs vev, and $g$ the coupling, then the low-energy effective action is an expansion in powers of $E / v$. However, Higgsed gauge fields have masses which scale as $g v$, and so for weak coupling $g$, generate $E /(g v)$ effects which can be stronger than loworder effective action terms. Put another way, $\mathrm{W}$ bosons are light relative to the natural mass scale defining the metric. As a result, the effective field theory arising in the infrared from a gauged sigma model often cannot be the same as a nonlinear sigma model. There can still be a moduli space, 
a metric on the moduli space, and many other features consistent with nonlinear sigma models (as happens with, e.g., Narain moduli spaces in toroidally compactified heterotic strings), but strictly speaking, the infrared limit of a low-energy effective gauged sigma model in four dimensions need not be physically equivalent to a nonlinear sigma model.

This result implies an issue of presentation-dependence in four-dimensional theories, that does not exist in two dimensions. In two dimensions, we identify universality classes of renormalization group flow with stacks: a given stack can have multiple presentations with different UV physical descriptions (a nonlinear sigma model, a gauged sigma model, an orbifold) which mathematically correspond to the same stack. Physically, it is conjectured that those different presentations lie in the same universality class, that the renormalization group "washes out" all details of the presentation, leaving physics that only depends upon the stack and not how it is described or presented. In particular, typically we are only interested in conformal field theories arising at endpoints of renormalization group flow, so the details of a physical presentation of a massive UV theory are irrelevant.

Part of the point of the observation on four-dimensional low-energy effective field theories is that the presentation-independence we enjoyed in two dimensions no longer applies in four dimensions. We can also see this from another perspective, involving the gauge kinetic terms. A sum over maps from a space into a stack presented as $[X / G]$ involves a sum over $G$ bundles with connection - a sum over $G$-gauge fields. In two dimensions, gauge kinetic terms are generated dynamically, so we could effectively ignore them and identify a nonlinear sigma model on $[X / G]$ with a $G$-gauged sigma model on $X$ - the sum over maps includes the sum over gauge fields, and the gauge kinetic term comes for free. By contrast, in four dimensions, gauge kinetic terms are not generated dynamically. We can describe some aspects of a $G$-gauged sigma model on $X$ with the stack $[X / G]$, but we do not get a gauge kinetic term automatically, the stack does not even determine a classical value of the gauge coupling. Hence, in four dimensions, merely specifying a stack does not uniquely determine the physics.

In two-dimensional cases, we associated stacks with universality classes of renormalization group flow. Instead, in four dimensions we will use stacks as "universal" objects from which various different physical presentations can be associated. The details of those presentations, the presentationdependence, will no longer be physically irrelevant, unlike two dimensions; nevertheless, some (not all) of the physics will be determined by the stack. It is in this sense that we will associate stacks with low-energy effective four-dimensional (gauged) sigma models and related theories. 
Before going on, let us summarize the circumstances under which gerbes will be physically meaningful in a four-dimensional theory. One way for gerbes to be physically meaningful in a four-dimensional theory is if the fourdimensional spacetime is topologically nontrivial, with nonzero $H^{2}(\mathbf{Z})$. In this case, one would have nontrivial nonperturbative sectors in the examples above. Just as in two dimensions, to uniquely define Higgs fields one would need to specify the precise bundle the field couples to, and that choice would be reflected in zero mode spectra, hence in anomalies and so forth. A second way gerbes can be physically meaningful is if there are massive non-invariant states. We have only argued this above in theories coupled to gravity; however, we shall also see examples later where some aspects of gerbes manifest even in four-dimensional theories on $\mathbf{R}^{4}$ not coupled to gravity. Both of these cases had analogues in two dimensions - for example, the theta-angle-periodicity argument in two dimensions relied on the existence of massive minimally charged states. We shall see examples of both cases in the next section.

Partly with an eye towards nonabelian applications we shall discuss later in this paper, let us summarize the conclusions of this and the previous section in the following slogan:

Perturbative physics is determined by the Lie algebra of the gauge group, but nonperturbative physics is determined by the Lie group, not just the algebra.

Just as an asymptotic series expansion does not uniquely determine the function it is expanding, so too does the perturbative physics not uniquely determine the nonperturbative physics.

In the rest of this paper, we will outline gerbes in four-dimensional field theories and string compactifications.

\section{Examples, duality in gerby moduli "spaces"}

Gerby moduli "spaces" 8 seem to appear in both four-dimensional field theory and in string compactifications, as we shall outline in this section. Briefly, a gerbe looks locally like a quotient by a trivially acting groupalthough the group acts trivially, both mathematics and, at least sometimes, physics nevertheless knows about the group action. As sigma models on

\footnotetext{
${ }^{8}$ Strictly speaking, if there is a gerbe structure, then the moduli "space" is actually a stack, not a space, but because the language of stacks is as yet unfamiliar to many physicists, we will call them "gerby spaces" in much of this paper.
} 
gerbes can be viewed as sigma models on spaces or effective quotients with a restriction on nonperturbative sectors, these are precisely the examples discussed recently in [7].

In this section we shall discuss examples of gerby moduli spaces appearing in both field and string theories, and also discuss how the gerbiness behaves under field and string theoretic dualities.

\subsection{Field theory}

At a purely mathematical level, it is easy to generate examples of fourdimensional field theories with gerbe structures over their moduli spaces. As the moduli space of a field theory is typically of the form $[V / G]$, where $V$ is a vector space spanned by matter vevs and $G$ is the gauge group, whenever any subgroup of $G$ acts trivially on all of the massless matter, mathematically one could associate a gerbe structure to that moduli space. For example, in Yang-Mills theory with adjoint matter, the maximal torus of the gauge group acts trivially on matter vevs. Thus, if $r$ is the rank of the gauge group, then in such theories there is formally a $U(1)^{r}$ gerbe structure generically. ${ }^{9}$ The physical content of that gerbe structure is another matter. Morally, if not literally, ${ }^{10}$ a sigma model on $U(1)^{r}$ gerbe ought to be a $U(1)^{r}$ gauge theory, which certainly arise in Yang-Mills theories with only adjoint matter.

However, we need a bit more structure (such as massive noninvariant matter, or a topologically nontrivial spacetime four-manifold) before we believe such gerbe structures are physically meaningful. In addition, in this paper we will focus on finite gerbe structures (corresponding to Deligne-Mumford stacks, rather than Artin stacks). In the examples we shall discuss in this section, the gerbe structure will arise by focusing on the center of the gauge group. If we return again to Yang-Mills theories with adjoint matter, this means we consider the gerbe structure on the moduli space arising from the fact that the center acts trivially on the matter.

\footnotetext{
${ }^{9}$ This stabilizer changes over the moduli space; for example, at the origin where all vevs vanish, the stabilizer is all of $G$. Since the stabilizer changes, this is not, strictly speaking, a gerbe, but rather is a more general stack, that only looks like various gerbes on specific strata.

${ }^{10} \mathrm{~A}$ stack with non-finite stabilizers is known as an Artin stack. The geometric interpretation of Artin stacks is somewhat more complicated than that of Deligne-Mumford stacks, which the analysis of $[10-16,19-21]$ focused on. In this paper we also almost exclusively focus on Deligne-Mumford stacks.
} 
Our first physical example will involve a topologically nontrivial spacetime four-manifold. Consider $\mathcal{N}=4$ supersymmetric theories arising in recent work on the geometric Langlands program [39]. There, one compactifies a four-dimensional $\mathcal{N}=4$ theory along a Riemann surface to get a two-dimensional theory, a nonlinear sigma model whose target space is the Hitchin moduli space on the compactification curve. The authors of [39] observed that said moduli space has a number of components. An alternative way of understanding that fact is to utilize the finite gerbe story outlined above. If we start with a $G$ gauge theory in four dimensions, then following the ansatz above, the moduli space of the four-dimensional theory (and hence the target of the compactified two-dimensional sigma model) has a $Z(G)$ gerbe structure, where $Z(G)$ denotes the center of $G$. Application of the decomposition conjecture of [14] to the two-dimensional sigma model on the gerbe then quickly reproduces the multiple component structure worked out more painfully by [39], as discussed in $[14,40]$.

One lesson of the example from geometric Langlands above is that these formal gerbe structures on moduli spaces do have physical content - the disconnectedness of the target of the two-dimensional sigma model is a consequence of a gerbe structure on the moduli space. That said, duality often does not preserve centers of gauge groups: for example, S-duality in $\mathcal{N}=4$ maps $S U(n)$ gauge theories to $S U(n) / \mathbf{Z}_{n}$ gauge theories. In effect, the center of the gauge group is being exchanged for extra characteristic classes, disconnectedness in the two-dimensional target moduli space. Hence, gerbe structures are not duality-invariant.

We are often used to moduli spaces being invariant under duality operations - this is, after all, one of the standard checks of a duality. What is going on here is that the underlying space is unchanged, only the automorphisms that are paired with the space are changing. Therefore, the number of flat directions, the geometry of the flat directions is unchanged, only the automorphisms differ. Since it is only the number and geometry of the flat directions that must necessarily be preserved by duality, the fact that gerbe structures change does not contradict duality.

Let us examine this $\mathcal{N}=4$ duality in greater generality. Geometric Langlands exchanges the center $Z(G)$ with the dual of $\pi_{1}\left({ }^{\mathrm{L}} G\right)$, where ${ }^{\mathrm{L}} G$ denotes the Langlands dual to $G$. The center $Z(G)$ encodes a gerbe structure, and $\pi_{1}\left({ }^{\mathrm{L}} G\right)$ describes how the moduli space breaks into components (indexed by a characteristic class in $\left.H^{2}\left(X, \pi_{1}\left({ }^{\mathrm{L}} G\right)\right)\right)$. We can see how $Z(G)$ and $\pi_{1}\left({ }^{\mathrm{L}} G\right)^{*}$ are exchanged as follows. Let $M$ denote the weight lattice of the Lie group $G$. It is a sublattice of the weight lattice of the corresponding Lie algebra, which we shall denote $\Lambda$. ( $M$ is determined by the representations of the Lie group, instead of the Lie algebra.) If we let $R$ denote the root lattice, 
then in general

$$
R \subseteq M \subseteq \Lambda .
$$

The action of Langlands duality is to dualize each of these three lattices:

$$
\begin{aligned}
R \mapsto{ }^{\mathrm{L}} R & \equiv \operatorname{Hom}(\Lambda, \mathbf{Z}), \\
M \mapsto{ }^{\mathrm{L}} M & \equiv \operatorname{Hom}(M, \mathbf{Z}), \\
\Lambda \mapsto{ }^{\mathrm{L}} \Lambda & \equiv \operatorname{Hom}(R, \mathbf{Z}),
\end{aligned}
$$

and it is straightforward to see from (4.1) that

$$
{ }^{\mathrm{L}} R \subseteq{ }^{\mathrm{L}} M \subseteq{ }^{\mathrm{L}} \Lambda .
$$

In this language, the center and $\pi_{1}$ of $G$ are determined by the lattices above, as follows:

$$
\begin{aligned}
Z(G) & =(M / R)^{*}=\operatorname{Hom}(M / R, \mathbf{Z}), \\
\pi_{1}(G) & =(\Lambda / M)^{*}=\operatorname{Hom}(\Lambda / M, \mathbf{Z})
\end{aligned}
$$

(the first equality comes from the fact that $Z(G)$ is the kernel of the adjoint action, whose weights generate the root lattice), which should make it clear that

$$
Z(G)=\pi_{1}\left({ }^{\mathrm{L}} G\right)^{*}, \quad \pi_{1}(G)=Z\left({ }^{\mathrm{L}} G\right)^{*} .
$$

In other words, Langlands duality exchanges the center of a group $G$ with (the dual of) $\pi_{1}$ of the Langlands dual group ${ }^{\mathrm{L}} G$.

The Hitchin moduli stack, the target of the two-dimensional sigma model, is a $Z(G)$-gerbe over a disconnected space with multiple components. One has different components corresponding to the fact that there is a characteristic class in $H^{2}\left(X, \pi_{1}(G)\right)$, and the components are indexed by the value of that characteristic class. The effect of Langlands duality is to exchange $Z(G)$ gerbiness with $\pi_{1}\left({ }^{\mathrm{L}} G\right)$ disconnectness (see, e.g., [41] for a more detailed discussion). One might ask if there is an alternative description as some $Z(G) \times Z\left({ }^{\mathrm{L}} G\right)$ gerbe over another space, giving a duality-invariant stack, but we are told [42] such a construction does not exist.

Let us next consider some examples of gerbe structures appearing in the field theories discussed in [43-46]. These papers discuss examples in which an $\mathcal{N}=1$ supersymmetric gauge theory with a gerbe structure on its moduli space is (Seiberg-)dual to another $\mathcal{N}=1$ supersymmetric gauge theory which has monopoles. The massive, non-invariant matter on the gerbe side 
is dual to the monopoles. Just as in the geometric Langlands story above, the gerbe structure is not preserved by duality.

The prototype for these examples is discussed in [44]. That paper argued that a Spin(8) gauge theory with $N_{f}$ fields in the $\boldsymbol{8}_{V}$ and one field in the $\boldsymbol{8}_{S}$ is dual to a chiral $S U\left(N_{f}-4\right)$ theory with a symmetric tensor and $N_{f}$ fields in the antifundamental representation. When the $\boldsymbol{8}_{S}$ is given a mass, the dual $S U\left(N_{f}-4\right)$ theory is Higgsed to $S O\left(N_{f}-4\right)$ with $N_{f}$ vectors. Moreover, that $S O\left(N_{f}-4\right)$ theory admits a monopole, since $\pi_{2}\left(S U\left(N_{f}-4\right) /\right.$ $\left.S O\left(N_{f}-4\right)\right)=\pi_{1}\left(S O\left(N_{f}-4\right)\right)=\mathbf{Z}_{2}$. The perturbatively massive spinor in the $\operatorname{Spin}(8)$ theory is dual to the monopole in the $S O\left(N_{f}-4\right)$ theory.

In the original (unHiggsed) dual pair, on neither side does the moduli space admit a gerbe structure: no part of the center of $\operatorname{Spin}(8)$ acts trivially on both $\boldsymbol{8}_{V}$ and $\boldsymbol{8}_{S}$, and the center of $S U\left(N_{f}-4\right)$ does not act trivially on the antifundamentals. After Higgsing, a $\mathbf{Z}_{2}$ subgroup of the center of Spin(8) acts trivially on the remaining $\boldsymbol{8}_{V}$ fields, hence that branch of the moduli space (formally) admits a $\mathbf{Z}_{2}$ gerbe structure. (Its dual still does not have a gerbe structure on its moduli space.)

The upshot is that we have two dual theories, one with a gerbe structure on its moduli space and a massive spinor, dual to a theory without a gerbe structure on its moduli space, but with a massive monopole instead. For example, a Wilson loop in the spinor representation of $\operatorname{Spin}(8)$ is mapped to the 't Hooft loop in the magnetic $\mathbf{Z}_{2}[45]$.

Just as in geometric Langlands, we see that gerbe structures are not preserved by duality. This interpretation is reiterated (albeit without explicitly naming a gerbe structure) in $[45$, Section 2,46$]$ in terms of screening effects, and further examples of the same general form are given in $[45,46]$.

For completeness, note that the presence of massive nonminimally charged matter plays an important role in this story, just as it did in two-dimensional examples of theories with gerbe structures.

\subsection{String theory}

Just as in field theory, one can also (formally) associate gerbe structures to various moduli spaces, whenever there is a subset of the low-energy gauge group that acts trivially on massless fields (and nontrivially on at least one massive field). In this section, we will outline examples of gerby moduli spaces appearing in string compactifications. 
The first example is the Narain moduli space of toroidally compactified heterotic string theories. Just as with Yang-Mills theories with adjoint matter, there are at least two natural ways to formally add a stack structure to such moduli spaces, both of which revolve around the fact that part of the Narain moduli space describes flat connections on a torus. If we take the moduli stack of flat $G$-connections to be

$$
\left[\operatorname{Hom}\left(\pi_{1}, G\right) / G\right],
$$

then we have a stack which along strata has variable gerbe structures along strata (though as the stabilizer varies across strata, it is not considered globally to be a gerbe, unless $G$ has a nontrivial center). For example, at the point on the moduli space where low-energy adjoint scalars vanish, one has a $G$ gerbe; at more nearly generic points, where only a maximal torus $T$ commutes with adjoint scalars, one has a $T$ gerbe. The mathematical interpretation of such structures is just as in the field theory discussion previously.

In the case of field theories, we observed that a different stack structure may have greater physical relevance, involving only finite centers of stabilizers rather than the entire stabilizer. This structure also varies across strata, giving rise along any one stratum to a variety of possible gerbe structures. Globally, the entire stack would have a $Z(G)$ gerbe structure, where $Z(G)$ is the center of $G$, which can be enhanced over various strata. In the case of geometric Langlands, this was the gerbe structure that gave rise to the disconnectedness of the target Hitchin moduli space of the two-dimensional theory.

Phrased more simply, ordinarily we think of toroidally compactified heterotic strings as having a Narain moduli space (or rather, more generally [47], a moduli space with several components, one of which is the Narain moduli space). Here we are observing that the Narain moduli space (and other components) carry additional structure, at least formally, namely that of a gerbe. The moduli stack of toroidally compactified heterotic strings is a gerbe over a stack with, in general, several components, one of which is the Narain moduli space, plus enhanced gerbe structures on various strata. (A more formal discussion of such phenomena in the context of Hitchin moduli spaces can be found in, e.g., [41].)

In the case of a $\operatorname{Spin}(32) / \mathbf{Z}_{2}$ heterotic string compactification, the moduli space of the toroidally compactified string theory generically (and formally) has a $\mathbf{Z}_{2}$ gerbe structure, since the center of $\operatorname{Spin}(32) / \mathbf{Z}_{2}$ is $\mathbf{Z}_{2}$. As described elsewhere, for such a gerbe structure to be meaningful for a theory on $\mathbf{R}^{4}$, we also need massive states which are not invariant under the group. 
In the present case, the $\operatorname{Spin}(32) / \mathbf{Z}_{2}$ heterotic string has at its first massive level states transforming in the spinor representation of $\operatorname{Spin}(32) / \mathbf{Z}_{2}$, which is not invariant under the $\mathbf{Z}_{2}$ [48, Section 6.3.1, 49, Section 2.3], exactly as needed for a gerbe description of the moduli space to be physically relevant.

On the 10-dimensional heterotic string worldsheet, this proposed $\mathbf{Z}_{2}$ gerbe structure on the CFT moduli space manifests itself as the quantum symmetry $^{11}$ [51] associated with the left-moving GSO analogue that defines the $\operatorname{Spin}(32) / \mathbf{Z}_{2}$ string in its RNS presentation. (The center of $\operatorname{Spin}(32)$ is $\mathbf{Z}_{2} \times \mathbf{Z}_{2}$, and the GSO analogue itself is responsible for the $\mathbf{Z}_{2}$ quotient in $\operatorname{Spin}(32) / \mathbf{Z}_{2}$. As the action of the center is being expressed on associated vectors, not on the group itself, it manifests in terms of orbifolds and quantum symmetries. There is a closely analogous story for the $\operatorname{Spin}(16) / \mathbf{Z}_{2}$ in $E_{8}$ and RNS constructions of $E_{8} \times E_{8}$ heterotic strings.) In particular, all of the massless 10-dimensional states arise from a left-moving NS sector, and the only charged states are adjoints; all of the left-moving $\mathrm{R}$ sector states are massive. The quantum symmetry leaves the left-moving NS sector states invariant and multiplies the left-moving $\mathrm{R}$ sector states by a phase, which matches the effect of the $\mathbf{Z}_{2}$ gerbe structure.

For completeness, let us also consider the string-dual type I theory in 10 dimensions. The gauge group of the type I string is $S O(32)$, different from that of its dual 10-dimensional heterotic string. We have seen that under dualities, gauge groups will change - this is a typical property of Langlands duality, for example. The massless spectrum is invariant under the $\mathbf{Z}_{2}$ center of $S O(32)$, suggesting a gerbe structure; however, all of the massive states are also invariant, as the perturbative spectrum of the type I string in 10 dimensions contains only symmetric and antisymmetric 2-tensors [49, Section 2.3], compatible in principle with a gauge group

$$
S O(32) / \mathbf{Z}_{2}=\operatorname{Spin}(32) /\left(\mathbf{Z}_{2} \times \mathbf{Z}_{2}\right) .
$$

For this reason, we do not identify a gerbe structure on the moduli space of compactified type I strings. Furthermore, in close analogy with our discussion of, e.g., [43-46] in the previous subsection, there exists a

\footnotetext{
${ }^{11}$ The symmetry we are describing leaves the NS sector states invariant, but multiplies the $\mathrm{R}$ sector states by a sign. A $\mathbf{Z}_{2}$ quantum symmetry leaves the untwisted sector invariant, and multiplies the twisted sector by a sign, which is consistent with the symmetry in this case if one remembers that we are using R, NS to describe states on the cylinder, but the quantum symmetry is defined by (un)twisted sectors on the complex plane, and the conformal transformation between the two exchanges R and NS sectors [50, Section 7.1].
} 
particle-type topological defect in type I string theory, arising from an element of $\pi_{8}(S O(32))$, which transforms as a spinor of the Lie algebra [52], the same property as a massive perturbative state in the dual heterotic $\operatorname{Spin}(32) / \mathbf{Z}_{2}$ theory.

Along special loci this gerbe structure can be enhanced, as expected on general grounds from our discussion of moduli stacks of flat connections. Consider Higgsing a toroidally compactified $E_{8} \times E_{8}$ heterotic string, for example. There is no gerbe structure over the entire moduli space (as $E_{8}$ has no center). Now Higgs one of the $E_{8}$ 's to a $\operatorname{Spin}(16) / \mathbf{Z}_{2}$ subgroup. As all of the adjoint-valued scalars in the theory are derived by dimensionally reducing a 10-dimensional gauge field, Higgsing the $E_{8}$ should lift all components of those scalars that are not adjoints under $\operatorname{Spin}(16) / \mathbf{Z}_{2}$. The center of $\operatorname{Spin}(16) / \mathbf{Z}_{2}$ is $\mathbf{Z}_{2}$, and it acts trivially on the adjoints, the surviving massless matter. However, it does not act trivially on all of the string modes. The adjoint representation of $E_{8}$ decomposes as

$$
248=120+128
$$

where $\mathbf{1 2 0}$ is the adjoint representation of $\operatorname{Spin}(16) / \mathbf{Z}_{2}$, and $\mathbf{1 2 8}$ is a spinor. By Higgsing the $E_{8}$ to $\operatorname{Spin}(16) / \mathbf{Z}_{2}$, we give a mass to the $\mathbf{1 2 8}$, which is not invariant under the center of $\operatorname{Spin}(16) / \mathbf{Z}_{2}$. Thus, we have, at low energies, a gauge group with nontrivial $\left(\mathbf{Z}_{2}\right)$ center that acts trivially on massless matter, but nontrivially on massive matter.

Note that we can construct examples with $\mathcal{N}=1$ supersymmetry in four dimensions and gerbe structures on their moduli spaces by compactifying a $\operatorname{Spin}(32) / \mathbf{Z}_{2}$ heterotic string on a nontrivial Calabi-Yau threefold. For simplicity, let us consider such a heterotic string compactification with the standard embedding.

One way to see the existence of the gerbe structure on the moduli space is from worldsheet considerations. Just as in the 10-dimensional theory, all of the massless states arise from left-moving NS sectors; the left-moving R sectors contribute only massive states. As a result, the quantum symmetry associated to the left-moving GSO analogue (which leaves left NS sectors invariant, but acts by a phase on left $\mathrm{R}$ sectors) leaves the massless states invariant, but acts by a phase on massive states.

We can also see the gerbe structure on the moduli space in the low-energy effective field theory. Consider for simplicity a $\operatorname{Spin}(32) / \mathbf{Z}_{2}$ heterotic string compactification on a nontrivial Calabi-Yau threefold with the standard 
embedding. The low-energy gauge group is ${ }^{12}$

$$
\frac{\operatorname{Spin}(26) \times U(1)}{\mathbf{Z}_{4}} .
$$

The $U(1)$ factor is typically anomalous and Higgsed via a four-dimensional version of the Green-Schwarz mechanism [54,55], closely related to a (field-dependent, hence not directly relevant to this paper) Fayet-Iliopoulos parameter. The remaining $\mathbf{Z}_{2}$ center of $\operatorname{Spin}(32) / \mathbf{Z}_{2}$ descends to part of the center of the group above, and the massless states are all invariant under this $\mathbf{Z}_{2}$, as the massless states all descend from invariant representations of $\operatorname{Spin}(32) / \mathbf{Z}_{2}$.

In the context of heterotic compactifications on elliptically fibered CalabiYau's, the moduli stack of $G$-bundles for a group $G$ has, in essence, directions corresponding to moduli of the spectral cover and directions corresponding to the moduli of a line bundle on the spectral cover. The latter has, at least formally, a $Z(G)$-gerbe structure. In the dual $\mathrm{F}$ theory compactification, such moduli dualize to moduli of $G$ flux, suggesting [42] that the moduli of

\footnotetext{
${ }^{12}$ We can compute this as follows. We are embedding $S U(3)$ into a $\operatorname{Spin}(6)=S U(4)$ subgroup, so we begin by observing that $\operatorname{Spin}(32)$ has the subgroup [53] [Appendix A]

$$
\frac{\operatorname{Spin}(26) \times \operatorname{Spin}(6)}{\mathbf{Z}_{2}}
$$

Since the center of both $\operatorname{Spin}(26)$ and $\operatorname{Spin}(6)$ is $\mathbf{Z}_{4}$, there is only one diagonally acting $\mathbf{Z}_{2}$ subgroup. We can describe the center of the group above as generated by $a, b$, subject to the relations $a^{4}=b^{4}=1, a^{2}=b^{2}$. Now, we want the $\operatorname{subgroup~of~} \operatorname{Spin}(32) / \mathbf{Z}_{2}$, and after taking the second $\mathbf{Z}_{2}$ quotient we could have either a $\mathbf{Z}_{2} \times \mathbf{Z}_{2}$ or $\mathbf{Z}_{4}$ quotient of $\operatorname{Spin}(26) \times$ Spin(6), corresponding to quotienting either $a^{2}$ or $a b$, respectively. We can distinguish them as follows. For simplicity, replace $\operatorname{Spin}(26)$ by $\operatorname{Spin}(6)$, to form subgroups of $\operatorname{Spin}(12)$, and use the fact that $\operatorname{Spin}(6)=S U(4)$, the $\mathbf{4}, \mathrm{Alt}^{3} \mathbf{4}=\overline{\mathbf{4}}$ are the spinor representations, and $\mathrm{Alt}^{2} \mathbf{4}=\mathbf{6}$ the vector. The $\mathbf{Z}_{2}$ quotient (originally of $\operatorname{Spin}(32)$, now $\operatorname{Spin}(12)$ ) should flip the sign of the $\mathbf{1 2}=(\mathbf{6}, \mathbf{1}) \oplus(\mathbf{1}, \mathbf{6})$, and preserve only one of the two spinor representations. Since $a, b$ both act by multiplying the 4 by a fourth root of unity, it is straightforward to check that $a^{2}$ leaves the vector representation invariant, whereas $a b$ flips the sign of the vector representation. (Both preserve only one spinor of Spin(12).) Thus, we should quotient by $a b$, and hence the correct subgroup of $\operatorname{Spin}(32) / \mathbf{Z}_{2}$ is
}

$$
\frac{\operatorname{Spin}(26) \times \operatorname{Spin}(6)}{\mathbf{Z}_{4}}
$$

Embedding $S U(3)$ into $S U(4)=\operatorname{Spin}(6)$ leaves us with the maximal commutant shown. We would like to thank A. Knutson for a useful discussion of this issue. 
$G$ fluxes has a gerbe structure. In fact, naively not only do moduli spaces of $\mathrm{F}$ theory compactifications admit gerbe structure, but at least sometimes there is evidence that duals to some heterotic string compactifications are $\mathrm{F}$ theory compactifications on gerbes [42]. Specifically, it has been observed [42] that the multisection structures appearing in [56] in the $\mathrm{F}$ theory duals of heterotic CHL strings have an alternative interpretation in terms of elliptic fibrations over $\mathbf{Z}_{2}$ gerbes. Part of the point is that a heterotic compactification on an elliptic fibration with multisection is described by a spectral cover in a gerbe over the relative Jacobian, together with a (possibly fractional, in a sense we describe later) line bundle over the restriction of the gerbe to the spectral cover. The $G$ fluxes then behave as a torsor under the appropriate Deligne cohomology group. We shall not pursue such $\mathrm{F}$ theory structures further here.

We do not expect such gerbe structures to always appear in CFT moduli spaces. For example, consider a heterotic $E_{8} \times E_{8}$ string compactified on a simply connected Calabi-Yau threefold, with the standard embedding. Although one has embedded an $S U(3)$ bundle, and $S U(3)$ has a nontrivial center $\left(\mathbf{Z}_{3}\right)$, it has been embedded into an $E_{8}$, which has no center. We can determine the existence of a gerbe structure by looking at the charged matter in the low-energy effective field theory. To get a gerbe structure, the matter would all have to be invariant under the center of the low-energy effective gauge group (in this case, $E_{6}$, with center $\mathbf{Z}_{3}$ ). Neither $\mathbf{2 7}$ 's nor $\overline{\mathbf{2 7}}$ 's are invariant under the center of $E_{6}$ [57], hence we do not expect to get a gerbe structure on the CFT moduli space, since there is no gerbe structure on the field theory moduli space.

More generally, it is worth emphasizing that many moduli spaces which do not have gerbe structures globally will still have gerbe structures on subvarieties. For a simple example, the quotient stack $\left[\mathbf{C}^{2} / \mathbf{Z}_{2}\right]$ looks like the quotient space $\mathbf{C}^{2} / \mathbf{Z}_{2}$ everywhere except at the origin, where there is a copy of the classifying stack $B \mathbf{Z}_{2}$ inserted, which mathematically desingularizes the quotient space. In that example, one has a $\mathbf{Z}_{2}$ gerbe over the origin (as $B \mathbf{Z}_{2}$ is a gerbe over a point), but nowhere else. We have already seen such structures in moduli stacks of flat connections, and they can also arise in moduli stacks of spaces: for example, the moduli stack of elliptic curves admits special points which are locally quotients, and so have finite gerbe structures. (The elliptic curves at those points have automorphisms not possessed by generic elliptic curves.) Sometimes (though not always) a gerbe structure at a subvariety on a moduli space will reflect an orbifold structure. For example, the moduli space of K3 surfaces contains a $\mathbf{Z}_{2}$ orbifold point, at which the $\mathrm{K} 3$ is represented by $\left[T^{4} / \mathbf{Z}_{2}\right]$. In this example, the orbifold structure on the moduli space reflects the quantum symmetry of the orbifold theory (though as already noted, this is not always the case). 
In passing, we should also mention that there may be further examples of string theories with gerby moduli spaces implicit in [58], which "geometrically engineers" four-dimensional theories with nonabelian gauge groups from type II compactifications on singular spaces.

\section{Topological defects and gerby moduli spaces}

Recall stable topological defects are classified by the homotopy groups of the moduli space $M$ : cosmic strings ${ }^{13}$ arise from $\pi_{1}(M)$, monopoles from $\pi_{2}(M)$, textures from $\pi_{3}(M)$. Homotopy groups can be defined for moduli stacks (see, e.g., [60] and references therein), and in particular for moduli spaces with gerbe structures, and are not quite the same as the homotopy groups of the underlying spaces. In this section we will outline such homotopy and discuss their potential application to topological defects.

Let us begin by outlining some pertinent facts about homotopy of gerbes. For $\mathcal{M}$ a $G$-gerbe ${ }^{14}$ over $M$, there is a homotopy long exact sequence

$$
\cdots \longrightarrow \pi_{n}(B G) \longrightarrow \pi_{n}(\mathcal{M}) \longrightarrow \pi_{n}(M) \longrightarrow \pi_{n-1}(B G) \longrightarrow \cdots
$$

In the sequence above, $B G$ denotes the "classifying stack" of $G$ (so named because of formal similarities with the classifying space of $G$ ). Technically, the classifying stack is defined as

$$
B G \equiv\left[\frac{\text { point }}{G}\right]
$$

If we think of a gerbe as being analogous to a fiber bundle, then the fibers are copies of $B G$. In terms of homotopy groups, it can be shown that $\pi_{n}(B G)=\pi_{n-1}(G)$. In particular, for finite $G, \pi_{1}(B G)=G$. (As maps from a space $X \rightarrow B G$ are defined by principal $G$-bundles over $X$, and principal

\footnotetext{
${ }^{13}$ For example, the stringy cosmic strings of [59] arise from the fact that $\pi_{1}$ of the moduli stack of elliptic curves is $S L(2, \mathbf{Z})$. (This stack should be distinguished from its Deligne-Mumford compactification. That compactification maps onto $S^{2}$, hence its homotopy groups have all of the complexity of the homotopy groups of $S^{2}$ and more [42].) However, the higher homotopy groups all vanish, so from compactifications on elliptic curves, the only topological defects one can get are stringy cosmic strings.

${ }^{14}$ In the special case that the gerbe is trivial, i.e., $\mathcal{M}=M \times B G=[M / G]$ for trivially acting $G, M$ is a $G$-bundle over $\mathcal{M}$, and so there is an additional long exact sequence
}

$$
\cdots \longrightarrow \pi_{n}(G) \longrightarrow \pi_{n}(M) \longrightarrow \pi_{n}(\mathcal{M}) \longrightarrow \pi_{n-1}(G) \longrightarrow \cdots .
$$


$G$-bundles over $S^{1}$ are classified by conjugacy classes in $G$, not elements of $G$, this also means we should be careful about interpreting $\pi_{1}$ as a group of maps from a circle.)

Now, let us apply the description above to cosmic strings, and discuss whether topological defects should be classified by homotopy groups of the gerbe or the underlying space.

Consider, for example, a moduli space with a $\mathbf{Z}_{n}$ gerbe structure, call it $\mathcal{M}$. If we denote the underlying (technically, "coarse") moduli space by $M$, then topological defects would ordinarily be computed by homotopy of $M$. The effect of the gerbe is to add a $B \mathbf{Z}_{n}$ fiber over each point of $M$. Over any point of $M$, therefore, is a copy of $B \mathbf{Z}_{n}$, which has $\pi_{1}=\mathbf{Z}_{n}$.

If topological defects are classified by homotopy groups of the moduli stack, not the underlying moduli space $M$, then we would get a cosmic string defined by a loop around $B \mathbf{Z}_{n}$ fibers, which may or may not be globally stable depending upon global properties ${ }^{15}$ of the gerbe. This would be some new type of cosmic string, as ordinary cosmic strings arise from $\pi_{1}(M)$. In this new type of cosmic string, the moduli space scalars would be unchanged as one walks around the string, except that the theory would undergo some $\mathbf{Z}_{n}$ gauge transformation around such a loop. Only massive noninvariant fields would see that gauge transformation.

Let us now turn to physical examples. The hypothetical cosmic string above sounds very similar to the $\mathbf{Z}_{n}$ cosmic string discussed in, e.g., [61, Section 4.2.2]. There, one has an $S U(2)$ gauge theory with a pair of tripletvalued Higgs fields which are required (by virtue of a potential term) to be orthogonal. Giving the first Higgs triplet a vev breaks $S U(2)$ to $U(1)$; giving the second an (orthogonal) vev breaks $U(1)$ to $\mathbf{Z}_{2}$. After both symmetry breakings have occurred, one has $\mathbf{Z}_{2}$ cosmic strings, as $\pi_{1}\left(S U(2) / \mathbf{Z}_{2}\right)=\mathbf{Z}_{2}$. In such a theory, the moduli space of possible Higgs vevs has a natural $\mathbf{Z}_{2}$ gerbe structure, and the cosmic strings described by [61] seem to naturally

\footnotetext{
${ }^{15}$ Suppose, for example, the gerby moduli space $\mathcal{M}$ is the nontrivial $\mathbf{Z}_{n}$ gerbe over $\mathbf{P}^{1}$ defined by taking two homogeneous coordinates $x, y$ to have weight $n$ under $\mathbf{C}^{\times}$. The space $S^{3}$ is a circle bundle over this gerbe, so we have a homotopy long exact sequence of the form

$$
\cdots \longrightarrow \pi_{n}\left(S^{1}\right) \longrightarrow \pi_{n}\left(S^{3}\right) \longrightarrow \pi_{n}(\mathcal{M}) \longrightarrow \pi_{n-1}\left(S^{1}\right) \longrightarrow \cdots
$$

In particular, since $\pi_{0}\left(S^{1}\right) \cong \pi_{0}\left(S^{3}\right)$ and $\pi_{1}\left(S^{3}\right)=0$, we have that $\pi_{1}(\mathcal{M})=0$, and so the gerbe $\mathcal{M}$ is simply connected. Thus, our hypothetical cosmic string would not be globally stable.
} 
coincide with the cosmic strings we have outlined above arising from homotopy of the gerbe. In fact, our homotopy considerations would appear to give a new perspective on the $\mathbf{Z}_{n}$ cosmic strings of [61], as they are discussed there only as homotopy of group cosets, and here we seem to have found the same structure in homotopy of a moduli stack.

Unfortunately, further analysis does not seem to bear out this perspective. One seeming counterexample arises in [62, Section 4.2]. That reference also describes $\mathbf{Z}_{n}$ cosmic strings, though in that case, the adjoints act primarily as spectators, and the cosmic string solution naturally involves winding of vevs of massless fundamentals, with a potential fixing their vevs to be nonzero. In the present case, for physical relevance of a gerbe structure, we need noninvariant fields, albeit massive noninvariant fields. If the noninvariant fields are massive, then its vev vanishes, and any sort of winding solution of the form outlined in [62, Section 4.2], unlikely.

Here is a more convincing counterexample. Consider an $S U(2)$ gauge theory containing only a single Higgs triplet, the $S U(2)$ would only be broken to $U(1)$, and although the resulting theory has monopoles (as $\pi_{2}(S U(2) /$ $U(1))=\mathbf{Z})$, it does not have cosmic strings (as $\left.\pi_{1}(S U(2) / U(1))=0\right)$. Thus, in this case, the homotopy of the gerbe gives a misleading result.

One potential fix to the counterexample above is to replace DeligneMumford stacks with more general Artin stacks (which are not required to have finite stabilizers). In the example above, an $S U(2)$ gauge theory with a single Higgs triplet, an Artin moduli stack would naturally have a $U(1)$ gerbe structure. Now, $\pi_{2}(B U(1))=\pi_{1}(U(1))=\mathbf{Z}$, so the same homotopy analysis of the gerbe would imply the existence of monopoles in this example, matching the physical result. For that matter, as $\pi_{1}(B U(1))=\pi_{0}(U(1))=0$, there is no prediction of cosmic strings, also matching the physics. On the other hand, as the gerbe structure would only see the unbroken part of the gauge group, not the original gauge group, it seems unlikely that the example above would generalize to give accurate results in other cases.

Our tentative conclusion is that, at least for Deligne-Mumford moduli stacks, the homotopy of the gerbe is misleading, the extra elements of $\pi_{1}$ that one encounters do not reflect physically meaningful new cosmic string solutions, and that topological defects should be counted by homotopy of the underlying space. This then begs the question of how to understand cosmic strings and other topological defects when the moduli space is a more complicated stack.

For completeness, let us also formally discuss higher defects in DeligneMumford stacks, though as already established, their physical relevance may 
not be significant. To be specific, consider the $\mathbf{Z}_{n}$ gerbe over $\mathbf{P}^{1}$ defined by taking two homogeneous coordinates $x, y$ to have weight $n$ under $\mathbf{C}^{\times}$, rather than weight 1 . Call this gerbe $\mathcal{M}$. We shall consider hypothetical monopoles arising from the moduli stack $\mathcal{M}$. From the long exact sequence for homotopy, we have

$$
\pi_{2}\left(B \mathbf{Z}_{n}\right) \longrightarrow \pi_{2}(\mathcal{M}) \longrightarrow \pi_{2}\left(\mathbf{P}^{1}\right) \longrightarrow \pi_{1}\left(B \mathbf{Z}_{n}\right) \longrightarrow \pi_{1}(\mathcal{G})
$$

Now, $\pi_{2}\left(B \mathbf{Z}_{n}\right)=0$, and it can be shown $\pi_{1}(\mathcal{M})=0$, so we have

$$
0 \longrightarrow \pi_{2}(\mathcal{M}) \longrightarrow \pi_{2}\left(\mathbf{P}^{1}\right) \longrightarrow \mathbf{Z}_{n} \longrightarrow 0
$$

Thus, the total number of stringy monopoles arising from this gerby moduli space would be countable, just as for an ordinary projective space, but note that not every monopole arising from $\mathbf{P}^{1}$ arises when the moduli stack is a gerbe over $\mathbf{P}^{1}$, closely mirroring the fact that in two-dimensional sigma models on gerbes there is a restriction on degrees of allowed maps.

We leave for future work a detailed discussion of global topological defects for more general moduli stacks. Our results here suggest that global gerbe structures may not be relevant, at least for Deligne-Mumford moduli stacks. It is possible that this is ultimately a reflection of subtleties in low-energy effective actions discussed in Section 3. We shall not attempt to address the relevance of homotopy of gerbe structures that exist only over subvarieties, or homotopy of Artin moduli stacks.

In passing, we should mention that [9, Section 4.2] speculated on the existence of cosmic strings of the form above in cases with gerby moduli spaces.

\section{Consistency conditions on classical supergravity}

In this section we will discuss consistency conditions on classical supergravities. We begin by reviewing results $[1,7,8]$ for the case that the moduli space is a smooth manifold, and then we generalize to smooth Deligne-Mumford stacks, focusing on gerbes over manifolds.

\subsection{Review of standard supergravity case}

First, let us recall the argument of Bagger and Witten [1] that the Kähler class of the moduli space of scalars of a supergravity theory is quantized, 
in the case that that moduli space is a smooth manifold. First, recall that across coordinate patches on the moduli space, the Kähler potential $K$ transforms as

$$
K \mapsto K+f+\bar{f},
$$

where $f$ is a holomorphic function of moduli, which must be accompanied by a rotation of the gravitino $\psi_{\mu}$ and the superpartners $\chi^{i}$ of the scalar fields on the moduli space:

$$
\chi^{\mathrm{i}} \mapsto \exp \left(+\frac{\mathrm{i}}{2} \operatorname{Im} f\right) \chi^{\mathrm{i}}, \quad \psi_{\mu} \mapsto \exp \left(-\frac{\mathrm{i}}{2} \operatorname{Im} f\right) \psi_{\mu}
$$

Consistency of the rotations (6.1) across triple overlaps (even within classical physics) implies that the $f$ 's define a line bundle with even $c_{1}$. If we denote that line bundle by $\mathcal{L}^{\otimes 2}$, then the gravitino is a spinor-valued section of $T X \otimes \phi^{*} \mathcal{L}^{-1}$, where $X$ is the four-dimensional low-energy effective spacetime and $\phi: X \rightarrow M$ the boson of the four-dimensional nonlinear sigma model on the compactification moduli space $M$, and that the fermions $\chi^{i}$ are spinor-valued sections of $\phi^{*}(T M \otimes \mathcal{L})$. Similarly, one shows that the superpotential is a holomorphic section of $\mathcal{L}^{\otimes 2}$, and, in order to have a positive-definite metric, $\mathcal{L}^{-2}$ (whose $c_{1}$ matches the cohomology class of the Kähler form) must be ample. ${ }^{16}$

The recent paper [8] extended the analysis of [1] to gauged group actions. If we gauge the action of some group $G$ on the target space of the nonlinear sigma model in the supergravity theory, then we have to lift that group action to the Bagger-Witten line bundle $\mathcal{L}^{\otimes 2}$ in order to define the gauging globally. We can see this explicitly in the supergravity gauge transformations. Under an infinitesimal group action

$$
\delta \phi^{i}=\epsilon^{(a)} X^{(a) i},
$$

\footnotetext{
${ }^{16}$ Specifically, positive-definiteness of the metric implies that every closed analytic subvariety of the moduli space $M$ has positive volume with respect to $c_{1}\left(\mathcal{L}^{-2}\right)$, i.e., for $Y \subset M$ closed of dimension $p$,

$$
\int_{Y} c_{1}\left(\mathcal{L}^{-2}\right)^{p}>0
$$
}

which is equivalent to $\mathcal{L}^{-2}$ being ample. 
where $X^{(a)}$ is a holomorphic Killing vector describing the infinitesimal group action, the superpartners $\chi^{\mathrm{i}}$, gaugino $\lambda^{(a)}$, and gravitino $\psi_{\mu}$ transform as

$$
\begin{aligned}
\delta \chi^{\mathrm{i}} & =\epsilon^{(a)}\left(\frac{\partial X^{(a) \mathrm{i}}}{\partial \phi^{j}} \chi^{j}+\frac{\mathrm{i}}{2} \operatorname{Im} F^{(a)} \chi^{\mathrm{i}}\right), \\
\delta \lambda^{(a)} & =f^{a b c} \epsilon^{(b)} \lambda^{(c)}-\frac{\mathrm{i}}{2} \epsilon^{(a)} \operatorname{Im} F^{(a)} \lambda^{(a)}, \\
\delta \psi_{\mu} & =-\frac{\mathrm{i}}{2} \epsilon^{(a)} \operatorname{Im} F^{(a)} \psi_{\mu},
\end{aligned}
$$

where $F^{(a)}=X^{(a)} K+\mathrm{i} D^{(a)}$ ( $K$ the Kähler potential), and $F^{(a)}$ is easily checked to be holomorphic. For real $\epsilon^{(a)}$, the Kähler potential undergoes a standard Kähler transformation

$$
\delta K=\epsilon^{(a)} F^{(a)}+\epsilon^{(a)} \bar{F}^{(a)},
$$

hence in the gauge transformations above, terms proportional to $\operatorname{Im} F^{(a)}$ are precisely encoding the Kähler transformations on fermions given in equation (6.1). Thus, the gauge-transformation terms proportional to $\operatorname{Im} F^{(a)}$ are encoding an infinitesimal lift of the group action to $\mathcal{L}$.

To define the gauge theory, we must extend the infinitesimal action encoded in supergravity to an action of the group, not just the Lie algebra. In general, lifts of group actions to line bundles need neither exist nor be unique. The existence issue provides a constraint on possible consistent supergravities. (Lack of) uniqueness is encoded in the Fayet-Iliopoulos parameter, as it is argued in [8] that implicit in the supergravity is the statement that the Fayet-Iliopoulos parameter determines different lifts of the action of $G$ to $\mathcal{L}^{\otimes 2}$. As such lifts are quantized, the Fayet-Iliopoulos parameter is necessarily quantized, and corresponds to an element of $\operatorname{Hom}(G, U(1))$ for $G$ the gauge group. Just as D-terms are understood in rigid supersymmetry in terms of symplectic quotients, the paper [8] argues that the structure above in supergravity can be understood in terms of "geometric invariant theory" quotients (see, e.g., [63-65]), the algebro-geometric analogue of symplectic quotients. In particular, in a geometric invariant theory quotient, the analogue of the Fayet-Iliopoulos parameter is quantized, because it is realized as a lift of a group action to a line bundle.

In the rest of this section we shall extend the analysis of $[1,8]$ to smooth Deligne-Mumford stacks, focusing on gerbes over manifolds. 


\subsection{Generalization to smooth Deligne-Mumford stacks}

The original work of Bagger-Witten [1] and follow-ups [8], reviewed above, only considered supergravity theories in which the moduli space is a smooth manifold. However, moduli spaces which are smooth manifolds are vanishingly rare - more typically, they have singularities and/or various stack structures, and a generalization of $[1,8]$ to such cases would be useful.

Formally, generalizing $[1,8]$ to moduli "spaces" that are smooth ${ }^{17}$ Deligne-Mumford stacks is very straightforward - the analysis of $[1,8]$ applies with only minimal modification. The main caveat is that specifying a "nonlinear sigma model on a stack" does not uniquely specify the physics; we must choose a presentation of the stack, and we could get different physics according to the choice. Put another way, there are multiple distinct physical theories, components of possibly multiple supergravities, that can be interpreted as a nonlinear sigma model on a single fixed stack. In four dimensions, we use stacks to provide a "universal" four-dimensional object for which any given physical realization corresponds to a presentation.

Let us outline the analysis in two different presentations:

- Deligne-Mumford stacks have coverings by open sets, so first consider a presentation in which the atlas is such a collection. Then, we can work patch-by-patch. Physically, this means we have a nonlinear sigma model on each open set, with perhaps a discrete gauge group. Just as for spaces, transformations of the Kähler potential across coordinate patches imply that there is a line bundle $\mathcal{L}$ over the moduli space, to which the gravitino $\psi_{\mu}$ and chiral superpartners $\chi^{i}$ couple. In other words, just as for the case that the moduli stack $M$ is a space, the gravitino is a spinor-valued section of $T X \otimes \phi^{*} \mathcal{L}^{-1}(X$ the four-dimensional spacetime, $\phi: X \rightarrow M)$, and the superpartners $\chi^{i}$ are spinor-valued sections of $\phi^{*}(T M \otimes \mathcal{L})$. The superpotential is a section of $\mathcal{L}^{\otimes 2}$. The Fayet-Iliopoulos parameter is a choice of lift of group action to $\mathcal{L}$, and such choices, possible values of the FayetIliopoulos parameter are elements of $\operatorname{Hom}(G, U(1))$ for $G$ the gauge group.

- Now, let us consider another presentation. Nearly all (see [12] for a discussion of exceptions) smooth Deligne-Mumford stacks can be

\footnotetext{
${ }^{17}$ Experts should note that since we are implicitly working over the complex numbers, "smooth" implies, for example, that there are no nonreduced scheme structures [42].
} 
presented as global quotients of ordinary smooth manifolds by (not necessarily finite) groups, whose actions need not be effective. To such presentations we can associate gauged supergravities, to which we can immediately apply [8]. To be specific, suppose the moduli stack is presented as $[Y / G]$ for some smooth manifold $Y$ and some group $G$, corresponding physically to a supergravity theory with moduli space $Y$ and gauged ${ }^{18} G$ action. In this case, the Bagger-Witten line bundle on the cover $Y$ with a $G$-equivariant structure (specified when one defines the gauge theory [8]) is equivalent to a (Bagger-Witten) line bundle on $[Y / G]$. Other results follow analogously. For example, in this presentation, quantization of the Kähler form on the stack $[Y / G]$ follows from both quantization of the Kähler form on $Y$ [1] and from quantization of Fayet-Iliopoulos parameters [8]. In all cases, generalizing Bagger-Witten [1] to this presentation intertwines the analyses and results of $[1,8]$. (The toy example of $[7]$ was realized by a presentation of this form.)

There exist more types of presentations of stacks (e.g., groupoid quotients), and so possibly more physical theories; in this paper, we shall discuss only the presentations above. As emphasized in Section 3, even in the IR these presentations can be physically distinct.

Regarding metric positivity, notions of ampleness and corresponding constraints on stacks are discussed in [60]; we assume, but have not carefully checked, that they are pertinent here.

In other words, formally, the results of $[1,8]$ carry over more or less immediately to smooth Deligne-Mumford stacks, at least in presentations of the form above. The only significant differences are as follows:

- A technical point is that cohomology of stacks more naturally lives in a different stack, the "associated inertia stack," not the stack itself. Thus, the analysis of [1] still implies that the cohomology class of the Kähler form on the moduli stack should match the cohomology class of $c_{1}$ of the Bagger-Witten line bundle on the stack, but although the Kähler form and Bagger-Witten line bundles themselves live on the stack, the cohomology lives in the associated inertia stack, and must be compared there. This adds no essential physical constraint.

- Because the stack is, roughly, a space with (finite) automorphisms, coordinate patches need match only up to (finite) automorphisms.

\footnotetext{
${ }^{18}$ The stack does not specify a classical gauge coupling; again, in four dimensions, we associate stacks to physics but not physics to stacks.
} 
Hence, for bundles on stacks, transition functions on triple overlaps need only close up to finite automorphisms. This means an honest bundle on a stack can be a "twisted" or "fractional" bundle on a space objects which are not bundles in the ordinary sense. (We shall define these below.) Put another way, there are more bundles on stacks than on underlying spaces, and many things on spaces that are not quite bundles, become honest bundles on stacks. Therefore, we need to carefully examine possible Bagger-Witten line bundles on stacks for possible physical subtleties.

Let us examine the second issue above, in the special case of smooth Deligne-Mumford stacks that have a (finite) gerbe structure over a smooth manifold. A twisted bundle on a space (see, e.g., [66-69]) is a bundle in which the transition functions close only up to a higher cocycle; schematically:

$$
g_{\alpha \beta} g_{\beta \gamma} g_{\gamma \alpha}=h_{\alpha \beta \gamma}
$$

for some Cech cocycle $h_{\alpha \beta \gamma}$, where the $g_{\alpha \beta}$ are transition functions. Consistency requires that the rank of a twisted bundle be related to the order of the cohomology element defined by $\left(h_{\alpha \beta \gamma}\right)$; since we are interested in line bundles, no nontrivial twisted bundles can contribute. Therefore, we need only consider the possibility that the Bagger-Witten line bundle might be a fractional line bundle.

To explain fractional line bundles, which will play a crucial role in this section, let us give an explicit example. Consider ${ }^{19}$ a $\mathbf{Z}_{k}$ gerbe on $\mathbf{P}^{n}$ defined by $n+1$ homogeneous coordinates of weight $k$, i.e.,

$$
\left[\frac{\mathbf{C}^{n+1}-0}{\mathbf{C}^{\times}}\right],
$$

where the $\mathbf{C}^{\times}$acts as

$$
\left(x_{0}, \ldots, x_{n}\right) \mapsto\left(\lambda^{k} x_{0}, \ldots, \lambda^{k} x_{n}\right)
$$

\footnotetext{
${ }^{19}$ This example could not arise physically because of anomalies. We give it here as a purely mathematical demonstration and explanation of fractional line bundles, no more.
} 
On this gerbe one can define line bundles with arbitrary weight under the $\mathbf{C}^{\times}$. For example, a line bundle of weight $m$ has total space $^{20}$

$$
\left[\frac{\left(\mathbf{C}^{n+1}-0\right) \times \mathbf{C}}{\mathbf{C}^{\times}}\right]
$$

where the $\mathbf{C}^{\times}$acts as

$$
\left(x_{0}, \ldots, x_{n} ; y\right) \mapsto\left(\lambda^{k} x_{0}, \ldots, \lambda^{k} x_{n} ; \lambda^{m} y\right) .
$$

When $m$ is divisible by $k$, this is the pullback of an honest line bundle on $\mathbf{P}^{n}$, namely $\mathcal{O}(m / k)$. More generally, the line bundle above on the gerbe is sometimes (ambiguously) denoted $\mathcal{O}(m / k)$ even when $m$ is not divisible by $k$. In such cases, one has a "fractional" line bundle. (See [16] for a more complete description of fractional line bundles.)

In passing, the properties of fractional branes at orbifold points are not unrelated to fractional bundles. Ultimately the reason for the relationship is that in an orbifold, there is a gerbe structure appearing over orbifold points, which has the effect of desingularizing the orbifold.

It should now be clear that these fractional line bundles on the gerbe are precisely what is being described in the example of [7], and more generally whenever one has "fractional Fayet-Iliopoulos parameters." If one were to pick a different physical presentation of the gerby moduli "space," say as a nonlinear sigma model with a restriction on nonperturbative sectors rather than as a gauged linear sigma model, then the line bundle $L$ of which the superpotential is a section would just be taken to be a fractional line bundle from the outset.

\footnotetext{
${ }^{20}$ Curiously, total spaces of fractional line bundles over gerbes often have the property that they are honest spaces with orbifolds, instead of gerbes, as is implicit in the expression given. Despite the existence of the orbifold structure along the zero section, one still has a notion of local trivializations; over the gerbe, the total spaces of the fractional bundles above have a local description of the form $U \times B \mathbf{Z}_{k} \times \mathbf{C}$ for $U$ an open patch on $\mathbf{P}^{n}$ and $B \mathbf{Z}_{k}$ the classifying stack of $\mathbf{Z}_{k}$. The point is that for any vector space $V$, the quotient $[V / G]$ is the same stack as the total space of a vector bundle of fiber $V$ over $B G$. The difference between the two descriptions might be described as distinguishing fibers over "gerby points" from fibers over "variety points": in the former case, one speaks of a vector bundle over $B G$, whereas in the latter, one speaks of $[V / G]$. Put another way, the (representable) projection map to the gerbe on $\mathbf{P}^{n}$ has two types of fibers: the fiber over a point of $\mathbf{P}^{n}$ is
}

$$
\left[\frac{\mathbf{C}_{k}^{\times} \times \mathbf{C}_{m}}{\mathbf{C}^{\times}}\right]
$$

(with subscripts indicating weights), whereas the fiber over a $B \mathbf{Z}_{k}$ is just $\mathbf{C}$. 
Now that we have explained fractional line bundles, let us return to our discussion of them as possible Bagger-Witten line bundles arising when the moduli stack possesses a (finite) gerbe structure, and discuss possible consistency conditions. We see two possibilities:

(1) One possibility is that allowed Kähler forms can have cohomology classes matching the (image of the) first Chern class of any line bundle on the gerbe, including any fractional line bundle.

(2) Another possibility is that allowed Kähler forms can have cohomology classes matching the (image of the) first Chern class only of line bundles which are pullbacks of line bundles on the underlying space - no fractional line bundles allowed.

The recent paper [7] argued the former case, that if the moduli "space" of the supergravity theory were actually a gerbe over an ordinary space then the quantization condition of Bagger-Witten should be modified, and fractional values of the Fayet-Iliopoulos parameter should be allowed. We shall now study this claim in detail.

Let us re-examine the example ${ }^{21}$ of a $U(1)$ gauge theory coupled to supergravity discussed in Section 3, in the spirit of [7]. In this theory, the supergravity moduli space is $\mathbf{C}^{2 n+2}$, and under the gauged $U(1)$, the fields have the following charges: $n$ fields $\phi_{i}$ of charge $k, n$ fields $\tilde{\phi}_{i}$ of charge $-k$, one field $\chi$ of charge +1 , one field $\tilde{\chi}$ of charge -1 . Furthermore, the fields of charge \pm 1 have mass $m$.

Restricting to the massless fields, the D-term condition has the form

$$
\sum_{i} k\left|\phi_{i}\right|^{2}-\sum_{i} k\left|\tilde{\phi}_{i}\right|^{2}=r
$$

where $r$ is the Fayet-Iliopoulos parameter. As discussed elsewhere [7,8], in supergravity $r$ is constrained to be an integer, so [7] observed that when we divide by the common factor of $k$, the D-term condition becomes

$$
\sum_{i}\left|\phi_{i}\right|^{2}-\sum_{i}\left|\tilde{\phi}_{i}\right|^{2}=r / k
$$

formally giving a fractional Fayet-Iliopoulos parameter (albeit normalized in such a way as to make that fact less explicit).

\footnotetext{
${ }^{21}$ The supersymmetric $\mathbf{C P}^{n}$ model would be marginally simpler to describe, but is also anomalous.
} 
Naively, the model above appears to describe a loophole in the analysis of Bagger-Witten [1], by allowing for fractionally quantized metrics. However, as discussed in Section 3, the infrared limit of a four-dimensional gauged sigma model (in effective field theory) need not be the same as a four-dimensional nonlinear sigma model. Thus, the example above is not describing a loophole in Bagger-Witten, as it does not RG flow to a theory of the form analyzed by Bagger-Witten. Rather, it is giving a quantization condition on a different theory than is considered by Bagger-Witten. (We would like to thank J. Distler for emphasizing this point to us.)

The physics above maps to a stack, a $\mathbf{Z}_{k}$ gerbe over a space $\mathbf{C}^{2 n} / / \mathbf{C}^{\times}$, and a fractional line bundle that extracts the "universal" aspects of the physics above. The fractional line bundle over the gerbe corresponds to the equivariant structure implicit in the choice of Fayet-Iliopoulos parameter. We cannot consistently construct a low-energy effective field theory by integrating out the Higgsed gauge field, and so there is no regime in which we can consistently talk about a fractionally quantized metric; however, we can nevertheless apply stacks to give a "universal" object encoding some essential aspects of the physics, and the gauge theory in question would be described mathematically by a fractional line bundle on a gerbe.

So far we have discussed the interpretation of certain choices of equivariant structures on Bagger-Witten line bundles. It remains to understand whether those choices that lead to fractional line bundles on gerbes are physically consistent.

In particular, let us examine the kinetic terms for the gravitino $\psi_{\mu}$ and superpartners $\chi^{I}$ more systematically. Recall the gravitino $\psi_{\mu}$ is a spinorvalued section of $T X \otimes \phi^{*} \mathcal{L}^{-1}$, and the fermions $\chi^{I}$ are spinor-valued sections of $\phi^{*}(T \mathcal{M} \otimes \mathcal{L})$, where $X$ is the four-dimensional low-energy effective spacetime, and $\phi: X \rightarrow \mathcal{M}$ is the bosonic map in the four-dimensional nonlinear sigma model in the supergravity. If the moduli space admits a gerbe structure, and the Bagger-Witten line bundle $\mathcal{L}$ is fractional, then there are some potential issues:

- First, fractional line bundles have no smooth (or even continuous) single-valued sections.

- Second, as noted earlier, seen as bundles over the underlying space, fractional bundles have orbifold singularities in their fibers, making a metric on those fibers potentially singular. As that metric appears in the fermion kinetic terms, this is potentially a hazard.

In principle, both of these problems are solved by the fact that if the moduli space $\mathcal{M}$ has a gerbe structure, then the path integral only sums over maps 
$\phi$ with degrees satisfying certain divisibility properties - this is one of the defining properties of a nonlinear sigma model on a gerbe. (That said, if the four-dimensional spacetime $X$ is $\mathbf{R}^{4}$, then as discussed before this matter is somewhat trivial, but let us describe the most general case here.) That same divisibility criterion ensures that $\phi^{*} \mathcal{L}$ is an honest line bundle, not anything fractional. As a result, even when $\mathcal{L}$ is fractional, the gravitino $\psi_{\mu}$ and fermions $\chi^{I}$ do exist as single-valued objects, coupling to honest bundles, with smooth fiber metrics.

In passing, let us mention another potential issue. If the SCFT moduli space admits a gerbe structure, and the Kähler form arises from a fractional line bundle, then there is an interesting structure on the worldsheet operators over SCFT moduli space (see [70] for a discussion for ordinary moduli spaces). Specifically, as we walk around the SCFT moduli space, some of the worldsheet operators (including the spectral flow operator) acquire phases from the (fractional) line bundle, and hence are necessarily multi-valued over the SCFT moduli space. This is at least odd, though not necessarily a physical contradiction. For example, the $S L(2, \mathbf{Z})$ transforms in monodromies on the $u$-plane in Seiberg-Witten theory tell us that the low-energy effective action there is really only globally defined on an $S L(2, \mathbf{Z})$-Riemann surface covering the $u$-plane, not the $u$-plane itself. The situation here is closely analogous.

As indirect consistency checks that these theories with fractional BaggerWitten line bundles are consistent, let us point out some closely related (and consistent) examples:

- One example occurs in two-dimensional $(0,2)$ SCFTs, describing heterotic strings on gerbes. If one compactifies a heterotic string on a gerbe with a fractional or twisted gauge bundle (i.e., a bundle on the gerbe that is not a pullback from the underlying space), the result looks like a sigma model on a space with a non-honest bundle, and a restriction on degrees of maps such that the pullbacks of non-honest bundles are honest. These will be discussed in detail in [16]. (Note the left-moving worldsheet fermions in this example are closely analogous to the four-dimensional gravitino and so forth we have been discussing - both couple to pullbacks of fractional bundles.) One way to construct examples is through asymmetric orbifolds, that act ineffectively on right-movers but effectively on left-movers. Examples can also be constructed in $(0,2)$ GLSMs, such as the (anomaly-free, fractional) bundle

$$
0 \longrightarrow \mathcal{E} \longrightarrow \mathcal{O}(1)^{\oplus 9} \longrightarrow \mathcal{O}(9) \longrightarrow 0
$$


over $\mathbf{P}_{[2,2,2,4]}^{3}[10]$, a $\mathbf{Z}_{2}$ gerbe over $\mathbf{P}_{[1,1,1,2]}^{3}[5]$. Other two-dimensional examples have been constructed by dimensional reduction of twisted four-dimensional $\mathcal{N}=2$ theories, as in [32]. These examples all seem to be consistent.

- It is perhaps worth observing that nonlinear sigma models on total spaces of fractional bundles are well-behaved. Consider a $(2,2)$ supersymmetric gauged linear sigma model describing

$$
\left[\frac{\left(\mathbf{C}^{2}-0\right) \times \mathbf{C}}{\mathbf{C}^{\times}}\right]
$$

where the $\mathbf{C}^{\times}$acts on $\left(\mathbf{C}^{2}-0\right)$ with weight 2 , and on $\mathbf{C}$ with weight 1 , say. This is the total space of the (fractional) line bundle $\mathcal{O}(1)$ over a $\mathbf{Z}_{2}$ gerbe on $\mathbf{P}^{1}$; it is also a modification of the exceptional set away from the weighted projective stack $\mathbf{P}_{[1,2,2]}^{2}$. This is a consistent $(2,2)$ supersymmetric theory.

- A four-dimensional gauge theory can also be constructed with closely analogous properties. Consider an $S U(n)$ gauge theory with matter in the fundamental of $S U(n)$. We can interpret this as the Feynman diagrams of $S U(n) / \mathbf{Z}_{n}$ gauge theory with a subset of the $S U(n) / \mathbf{Z}_{n}$ instantons (omitting fractional instantons), restricted so as to make the fundamental matter always well-defined. (We cannot precisely call this an $S U(n) / \mathbf{Z}_{n}$ gauge theory with a restriction on instantons, because the $S U(n) / \mathbf{Z}_{n}$ gauge transformations are not well-defined on the matter fields. For this reason, both the $S U(n)$ and the $S U(n) / \mathbf{Z}_{n}$ gauge theories obey cluster decomposition.) After all, perturbatively an $S U(n)$ and $S U(n) / \mathbf{Z}_{n}$ gauge theory are identical (same Lie algebra, same Lagrangian, same Feynman diagrams), the difference between the data given is that an $S U(n) / \mathbf{Z}_{n}$ has additional ("fractional") instantons not present in the $S U(n) / \mathbf{Z}_{n}$ theory. One could imagine splitting an $S U(n)$ instanton into $S U(n) / \mathbf{Z}_{n}$ instantons, but if one does so, one would have to introduce topological defects in order to allow the matter in the fundamental representation to be well-defined globally.

This last example perhaps best exemplifies the slogan

Perturbative physics is determined by the Lie algebra of the gauge group, but nonperturbative physics is determined by the Lie group, not just the algebra.

mentioned in Section 3. 
Our tentative conclusion is that "fractional Fayet-Iliopoulos parameters" are consistent in supergravity theories in which the moduli stack is a gerbe, and are a reflection of stacky subtleties arising in more general supergravity theories. One should be careful about asserting that this implies a loophole in Bagger-Witten's old quantization result [1], as the infrared limit of a four-dimensional gauged sigma model need not be the same as a nonlinear sigma model. We leave a more detailed analysis of consistency conditions in supergravity theories with moduli stacks to future work.

\section{Conclusions}

In this paper we have reviewed recent discussions of quantization of the Fayet-Iliopoulos parameter in supergravity theories. We began this paper by reviewing previous work on two-dimensional theories with restrictions on nonperturbative sectors - equivalently, sigma models on gerbes - and more general aspects of two-dimensional sigma models on stacks, followed by a discussion subtleties appearing in four-dimensional analogues. We gave examples in both field and string theory of models with gerbe structures on their moduli spaces, and discussed the action of duality. We discussed global topological defects when the moduli space is a stack, focusing on stacks that are gerbes over smooth manifolds. We then discussed consistency conditions on classical supergravity theories for moduli spaces that are smooth Deligne-Mumford stacks, after reviewing the state-of-the-art for smooth manifolds.

In the text we listed a number of interesting possible follow-ups. Another direction that would be interesting to pursue is sigma model anomalies, in the sense of Moore-Nelson [71-73], in cases where the target space is a gerbe or other stack.

Yet another direction concerns deformation issues. Briefly, stacks and underlying spaces do not always admit the same deformations. To illustrate the principle, consider a local quotient stack structure resolving an orbifold singularity on a Calabi-Yau. (Moduli spaces are typically not Calabi-Yau, but this will provide a simple example of the deformation theory issue.) Although quotient spaces often admit Calabi-Yau blowups, corresponding quotient stacks do not. (In string compactifications on stacks, this leads to an apparent mismatch in moduli which was discussed in [12].) Notions of blowup still exist, but are usually not Calabi-Yau. For moduli stacks appearing in field theory and string theory, then, a natural question to ask is whether the existence of a quotient stack structure "resolving" an orbifold 
singularity on the moduli space reflects any obstruction to resolution or deformation of the singularity. It would be interesting to understand if this deformation-theoretic mismatch had any applications in either field or string theory.

There are several other potential applications of such gerbe and stack structures in field theory moduli spaces that we can imagine. For example, it would be interesting to understand whether "stacky" resolutions of quotient singularities on moduli spaces, i.e., $\left[\mathbf{C}^{n} / G\right]$ versus $\mathbf{C}^{n} / G$, convey any additional information about the theory, such as properties of light particles. It would also be interesting if gerbe structures could be used to help disentangle confusing potential Seiberg duals. Examples of such are discussed in, for example, [74], and there is a gerbe structure on some of the moduli spaces of the field theories discussed there. Similarly, it would be interesting to understand the three-dimensional "mirrors" [75] to theories with nonminimally charged electrons. In two dimensions, such mirrors turned out to involve either discrete-valued fields [13] or, equivalently, disconnected targets [14].

It would also be interesting to understand if the ideas in this paper could be applied to understand the distinctions between $S U(2)$ and $S O(3)$ Donaldson and related mathematical invariants, see, e.g., [76] and references therein.

\section{Acknowledgments}

Some of the ideas in this paper have germinated over the last decade and been discussed with numerous people, more than we can list here. In particular, we would like to thank J. Distler for numerous discussions of cluster decomposition and supergravity moduli space issues, A. Knutson for many discussions of group theory pertinent to heterotic strings, T. Pantev for many years of discussions and collaboration on strings propagating on gerbes and stacks, and M. Strassler for insights and references on four-dimensional gauge theoretic analogues provided during a visit to the University of Washington in spring 2005. We would also like to thank P. Argyres, A. Shapere, and M. Unsal for more recent useful conversations.

The work of S.H. was supported by the WorldPremier International Research Center Initiative, MEXT, Japan, and by a Grant-in-Aid for Scientific Research (22740153) from the Japan Society for Promotion of Science (JSPS). E.S. was partially supported by NSF grants DMS-0705381 and PHY-0755614. 


\section{Appendix A. Four-dimensional decomposition conjecture}

In this appendix, we will discuss a four-dimensional analogue of the decomposition conjecture for two-dimensional CFTs discussed in [14]. This will arise via restricting four-dimensional instantons (mathematically, $c_{2}$ 's, not $c_{1}$ 's), and so will not be directly relevant for the gerbes studied elsewhere in this paper.

Consider a four-dimensional SCFT obtained from a gauge theory, e.g., $\mathcal{N}=4 S U(n)$ SYM, or $\mathcal{N}=2 S U(n)$ SYM with $2 n$ hypermultiplets in the fundamental, or one of the $\mathcal{N}=1$ SCFTs.

In that gauge theory, restrict the nonperturbative sector to instantons of degree divisible by $k$. Note that the resulting theory will not be associated to gerbes - we are here imposing a restriction on Pontryagin classes of bundles, visible to four-dimensional theta angles, whereas gerbe structures would only affect analogues of the first Chern class. This theory automatically violates cluster decomposition, by Weinberg's ancient argument; we shall describe how it can be written formally as a sum of other theories with rotating theta angle.

In this theory, since the instantons have degrees divisible by $k$, the Chern-Simons vacua split into $k$ separate sets. The allowed instantons define tunnelling only between Chern-Simons vacua within the same set. In this fashion, one recovers $k$ separate zero-energy ground states. Under the assumption that when the gauge field is extended flatly in extra dimensions, the Chern-Simons number is cobordism invariant, the Chern-Simons number is conserved modulo $k$.

Using the state-operator correspondence for conformally invariant theories, one can build $k$ different zero-energy states, which for the reasons above obey the same multiplicative rules as twist fields in the two-dimensional theories discussed in [14], and hence can be used to define projection operators.

Thus, we conjecture that the four-dimensional SCFT above with theta angle $\theta$ can be decomposed into a sum of $k$ SCFTs, and we further conjecture that those $k$ SCFTs are copies of the SCFT with theta angles $\theta+2 \pi n / k$ for $n=0, \ldots, k-1$, where $\theta$ has period $2 \pi$ in the theory where instanton number 1 configurations are allowed. (This sum has the effect of cancelling out gauge field configurations in the path integral whose instanton degrees are not multiples of $k$.)

For the two-dimensional decomposition conjecture pertinent to sigma models on gerbes, there is now abundant evidence, including all-genera 
partition function computations in orbifold examples [14], checks in mirror symmetry and quantum cohomology [14], applications to gauged linear sigma models [15], and now checks of predictions for Gromov-Witten invariants [33-38]. By contrast, in the four-dimensional case above, we have no independent evidence, no examples, only the arguments above.

\section{Appendix B. Two-dimensional BF theory and cluster decomposition}

In this section we will examine $B F$ theory in two dimensions, as an example of a manifestly local theory that does not obey cluster decomposition. Let $B$ be a circle-valued scalar, i.e., identified under $B \mapsto B+2 \pi$. Let $A$ be an abelian gauge field with the usual gauge transformation, so that locally

$$
\begin{aligned}
& A \mapsto A+d \chi, \\
& F \equiv d A,
\end{aligned}
$$

where $\chi$ is a circle-valued gauge parameter: $\chi \equiv \chi+2 \pi$. Then the field strength $F$ then satisfies the Dirac quantization condition,

$$
\int F \in 2 \pi \mathbf{Z}
$$

The action for $B F$ theory is

$$
S=\frac{k}{2 \pi} \int B F
$$

and the Euclidean action is

$$
S_{\mathrm{E}}=\frac{\mathrm{i} k}{2 \pi} \int B F .
$$

This theory is simple enough that it can be solved exactly and explicitly. To do this we solve for the dimension of the Hilbert space of states on a spatial slice $S^{1}$ and for the action of the operator algebra on that Hilbert space. The particular point to which we draw attention is the absence of cluster decomposition: inside the local operator algebra of the theory is a pair of local operators $\mathcal{O}_{ \pm 1}$ that disobey the condition for cluster decomposition, in the sense that

$$
\lim _{x \rightarrow \infty}\left\langle\mathcal{O}_{1}(x) \mathcal{O}_{-1}(0)\right\rangle \neq \lim _{x \rightarrow \infty}\left\langle\mathcal{O}_{1}(x)\right\rangle\left\langle\mathcal{O}_{-1}(0)\right\rangle
$$




\section{B.1 Hilbert space of the BF theory}

First, we compute the overall dimension of the Hilbert space of states on $S^{1}$. To do this, we compute the partition function on a spatial circle at finite temperature $\beta^{-1}$ :

$$
Z(\beta)=\sum_{k} \exp \left\{-\beta E_{k}\right\}
$$

Since the two-dimensional metric does not appear in the $B F$ action, we expect that the theory is topological and that the energies $E_{k}$ should all vanish identically, and that the partition function $Z(\beta)$ is therefore independent of $\beta$. We will see that this is indeed the case.

To compute the partition function at finite temperature $\beta^{-1}$, we perform the path integral in Euclidean signature with Euclidean time compactified with a radius of $r_{2} \equiv \frac{\beta}{2 \pi}$. We also compactify the spatial direction with radius $r_{1}$, so that the Hilbert space becomes manifestly separable. We have then reduced the finite-temperature partition function to a path integral over a discretely infinite set of variables, the Fourier modes of the $B$ field and the $U(1)$ gauge connection. The path integral over the nonzero modes is purely Gaussian, and can be performed straightforwardly so long as we divide appropriately by the measure for the local $U(1)$ gauge group. The path integral over the zero modes we perform separately.

\section{B.2 Path integral measure on a finite torus}

Path integrals in finite volume require a bit of care in order to get the overall normalization correct - we mostly follow the method of [77], deviating from the presentation there only in details particular to the application here.

Define the measure - for the gauge group, the gauge field, and the $B$ field - as in [77], in a local way. To do this, decompose the fields and the gauge parameter into normal modes:

$$
\begin{aligned}
A_{i}(x) & \equiv \sum_{M} a^{(M)} \phi_{i,(M)}(x), \\
B(x) & \equiv \sum_{N} b^{(N)} \phi_{(N)}(x) \\
\chi(x) & \equiv \sum_{N} c^{(N)} \phi_{(N)}(x)
\end{aligned}
$$


where $\phi_{(N)}$ is a set of unit-orthonormalized real eigenfunctions of the scalar Laplacian, and $\phi_{i,(N)}$ is a set of unit-orthonormalized real vector eigenfields of the vector Laplacian:

$$
\begin{aligned}
& \int d^{2} x \sqrt{g} \phi_{(N)}(x) \phi_{\left(N^{\prime}\right)}(x)=\delta_{N^{\prime} N}, \\
& \int d^{2} x \sqrt{g} g^{i j} \phi_{i,(M)}(x) \phi_{j,\left(M^{\prime}\right)}(x)=\delta_{M^{\prime} M},
\end{aligned}
$$

and the $a_{(M)}, b_{(N)}, c_{(N)}$ are mode amplitudes, the Fourier transforms of the dynamical fields. Then define the path integral measures

$$
\begin{aligned}
D A & \equiv \prod_{(M)} d a^{(M)}, \\
D B & \equiv \prod_{(N)} d b^{(N)}, \\
D \chi & \equiv \prod_{(N)} d c^{(N)},
\end{aligned}
$$

with unit normalization.

Concretely, for the torus, we can let $N$ run over the values 0 and ([p], re), where $([p], \mathrm{im})$. Here, the symbol $[p]$ represents a pair $\left\{p_{i},-p_{i}\right\}$ of equal and opposite nonzero momenta obeying appropriate quantization conditions, and re and im represent the real and imaginary parts of the mometum eigenfunction.

As for $M$, we let it run over nonzero modes $([p], \mathrm{re}, \perp),([p], \mathrm{im}, \perp),([p]$, re, $\|)$, and $([p], \mathrm{im}, \|)$, and also over zero modes labelled $(0, I)$. Here $[p]$ means the same as it does for the scalar eigenmodes, i.e., a pair of equal and opposite nonzero momenta on the two-torus, and re and im represent the real and imaginary parts of a plane wave. The symbols $\perp$ and $\|$ denote the transverse and longitudinal polarizations for the nonzero modes, and the $I$ labelling the zero modes (Wilson lines) runs over the two directions of the torus.

\section{B.3 Path integral over nonzero modes}

Let us now perform the path integral over nonzero modes. For a given pair $[p]$, we have two multiplicative contributions to $Z\left(r_{1}, r_{2}\right)$ : First, we have the Gaussian path integral over the nonzero modes $b_{([p], \mathrm{re})}, b_{([p], \mathrm{im})}$ and 
$a_{([p], \mathrm{re}, \perp)}, a_{([p], \mathrm{im}, \perp)}$. And then, we also have the Jacobian determinant of the gauge transformation of $a_{([p], \mathrm{re}, \|)}, a_{([p], \mathrm{im}, \|)}$ by $c_{([p], \mathrm{re})}, c_{([p], \mathrm{im})}$. First, we combine the real and imaginary parts of the plane waves into the natural complex combinations:

$$
\begin{aligned}
\Phi_{i,([p], \perp)} & \equiv \frac{1}{\sqrt{2}}\left(\phi_{i,([p], \mathrm{re}, \perp)}+\mathrm{i} \phi_{i,([p], \mathrm{im}, \perp)}\right), \\
\Phi_{i,([p], \|)} & \equiv \frac{1}{\sqrt{2}}\left(\phi_{i,([p], \mathrm{re}, \|)}+\mathrm{i} \phi_{i,([p], \mathrm{im}, \|)}\right) \\
\Phi_{[p]} & \equiv \frac{1}{\sqrt{2}}\left(\phi_{([p], \mathrm{re})}+\mathrm{i} \phi_{([p], \mathrm{im})}\right), \\
\mathbf{a}_{([p], \perp)} & \equiv \frac{1}{\sqrt{2}}\left(a_{([p], \mathrm{re}, \perp)}+\mathrm{i} a_{([p], \mathrm{im}, \perp)}\right), \\
\mathbf{a}_{([p], \|)} & \equiv \frac{1}{\sqrt{2}}\left(a_{([p], \mathrm{re}, \|)}+\mathrm{i} a_{([p], \mathrm{im}, \|)}\right), \\
\mathbf{b}_{[p]} & \equiv \frac{1}{\sqrt{2}}\left(b_{([p], \mathrm{re})}+\mathrm{i} b_{([p], \mathrm{im})}\right), \\
\mathbf{c}_{[p]} & \equiv \frac{1}{\sqrt{2}}\left(c_{([p], \mathrm{re})}+\mathrm{i} c_{([p], \mathrm{im})}\right) .
\end{aligned}
$$

In terms of the complex combinations above, the measure is

$$
\begin{aligned}
d a_{\mathrm{re}} d a_{\mathrm{im}} & =2 d^{2} \mathbf{a}=2 d(\operatorname{Re} \mathbf{a}) \wedge d(\operatorname{Im} \mathbf{a}), \\
d b_{\mathrm{re}} d b_{\mathrm{im}} & =2 d^{2} \mathbf{b}=2 d(\operatorname{Re} \mathbf{b}) \wedge d(\operatorname{Im} \mathbf{b}), \\
d c_{\mathrm{re}} d b_{\mathrm{im}} & =2 d^{2} \mathbf{c}=2 d(\operatorname{Re} \mathbf{c}) \wedge d(\operatorname{Im} \mathbf{c}),
\end{aligned}
$$

where we have suppressed the indices $[p], \perp$, and $\|$, and we have used the standard convention

$$
d^{2} \mathbf{z} \equiv \frac{\mathrm{i}}{2} d \mathbf{z} \wedge d \overline{\mathbf{z}}=d(\operatorname{Re} \mathbf{z}) \wedge d(\operatorname{Im} \mathbf{z})
$$

for the measure on a complex variable $\mathbf{z}$.

The expansion of $A_{i}(x), B(x)$, and $\chi(x)$ in eigenmodes takes the form

$$
\begin{aligned}
A_{i}(x)= & (\text { zero modes })+\sum_{[p]} \mathbf{a}_{([p], \perp)} \Phi_{i,([p], \perp)}+\mathbf{a}_{([p], \|)} \Phi_{i,([p], \|)} \\
& +\mathbf{a}_{([p], \perp)}^{*} \Phi_{i,([p], \perp)}^{*}+\mathbf{a}_{([p], \|)}^{*} \Phi_{i,([p], \|)}^{*},
\end{aligned}
$$




$$
\begin{aligned}
& B(x)=(\text { zero mode })+\sum_{[p]} \mathbf{b}_{[p]} \Phi_{[p]}+\mathbf{b}_{[p]}^{*} \Phi_{[p]}^{*}, \\
& \chi(x)=(\text { zero mode })+\sum_{[p]} \mathbf{c}_{[p]} \Phi_{[p]}+\mathbf{c}_{[p]}^{*} \Phi_{[p]}^{*} .
\end{aligned}
$$

The orthonormality conditions for the complex normal modes are

$$
\begin{aligned}
& \int d^{2} x \sqrt{g} \Phi_{[p]}^{*} \Phi_{\left[p^{\prime}\right]}=\int d^{2} x \sqrt{g} g^{i j} \Phi_{i,([p], \perp)}^{*} \Phi_{j,\left(\left[p^{\prime}\right], \perp\right)} \\
& =\int d^{2} x \sqrt{g} g^{i j} \Phi_{i,([p], \|)}^{*} \Phi_{j,\left(\left[p^{\prime}\right], \|\right)}=\delta_{[p],\left[p^{\prime}\right]}, \\
& \int d^{2} x \sqrt{g} g^{i j}\left(\Phi_{i,([p], \perp)}^{*} \Phi_{j,\left(\left[p^{\prime}\right], \|\right)}+\Phi_{i,\left(\left[p^{\prime}\right], \|\right)}^{*} \Phi_{j,([p], \perp)}\right) \\
& =\int d^{2} x \sqrt{g} g^{i j} \Phi_{i,([p], \perp)} \Phi_{j,\left(\left[p^{\prime}\right], \|\right)}=0, \\
& \int d^{2} x \sqrt{g} \Phi_{[p]} \Phi_{\left[p^{\prime}\right]}=\int d^{2} x \sqrt{g} g^{i j} \Phi_{i,([p], \perp)} \Phi_{j,\left(\left[p^{\prime}\right], \|\right)}=0 .
\end{aligned}
$$

and the transversality conditions are

$$
g^{i j} \partial_{i} \Phi_{j,([p], \perp)}=\epsilon^{i j} \partial_{i} \Phi_{j,([p], \|)}=0 .
$$

A natural choice for the normal modes is

$$
\begin{aligned}
\Phi_{[p]} & =\sqrt{\frac{1}{\mathrm{vol}_{2}}} \exp \{\mathrm{i} p \cdot x\}, \\
\Phi_{i,([p], \perp)} & =\sqrt{\frac{1}{\mathrm{vol}_{2}}} \sqrt{\frac{|g|}{g^{m n} p_{m} p_{n}}} \epsilon_{i k} g^{k l} p_{l} \exp \{\mathrm{i} p \cdot x\}, \\
\Phi_{i,([p], \|)} & =\sqrt{\frac{1}{\mathrm{vol}_{2}}} \sqrt{\frac{1}{g^{m n} p_{m} p_{n}}} p_{i} \exp \{\mathrm{i} p \cdot x\} .
\end{aligned}
$$

Now we want to write the Euclidean action in terms of the complex normal modes. The action is of course a topological invariant, but since the normalization conditions of the normal modes are written in terms of the metric, it is useful to write the action as

$$
S_{\mathrm{E}}=+\frac{\mathrm{i} k}{2 \pi} \int d^{2} x \sqrt{|g|} \frac{\epsilon^{i j}}{2 \sqrt{|g|}} B F_{i j}
$$


In terms of the complex normal modes the action is

$$
\begin{aligned}
S_{\mathrm{E}} & =\sum_{[p]} S_{[p]}, \\
S_{[p]} & \equiv+\frac{k}{2 \pi}|p|\left(\mathbf{b}_{[p]} \mathbf{a}_{([p], \perp)}^{*}-\mathbf{b}_{[p]}^{*} \mathbf{a}_{([p], \perp)}\right) \\
& =\left(\mathbf{b}_{[p]}^{*} \mathbf{a}_{([p], \perp)}^{*}\right) \mathcal{O}\left(\begin{array}{c}
\mathbf{b}_{[p]} \\
\mathbf{a}_{([p], \perp)}
\end{array}\right),
\end{aligned}
$$

where $\mathcal{O}$ is the anti-Hermitean matrix

$$
\mathcal{O} \equiv-\frac{\mathrm{i} k|p|}{2 \pi} \cdot \sigma^{2}, \quad|p| \equiv \sqrt{g^{i j} p_{i} p_{j}} .
$$

Since the operator $\mathcal{O}$ has imaginary eigenvalues, we can define the path integral by the prescription

$$
\mathcal{O} \rightarrow \mathcal{O}+\epsilon|p|^{2} \cdot \mathbf{1}_{2 \times 2}
$$

and let $\epsilon \rightarrow 0^{+}$be a positive real parameter approaching zero from above. With this definition the action is still local and reduces in the $\epsilon \rightarrow 0^{+}$limit to the undeformed action, but the path integral over each set of modes is convergent.

For an operator $\mathcal{O}$ with positive-definite real part, the Gaussian path integral over a vector of complex variables $\mathcal{A}$ is given by

$$
\int \prod_{(N)}\left(2 d^{2} \mathcal{A}_{(N)}\right) \exp \left\{-\mathcal{A}^{\dagger} \mathcal{O} \mathcal{A}\right\}=\left[\operatorname{det}\left(\frac{\mathcal{O}}{2 \pi}\right)\right]^{-1},
$$

so, letting

$$
\begin{aligned}
\mathcal{A} & \equiv\left(\begin{array}{c}
\mathbf{b}_{[p]} \\
\mathbf{a}_{([p], \perp)}
\end{array}\right), \\
\mathcal{O} & \equiv-\frac{\mathrm{i} k|p|}{2 \pi} \sigma^{2},
\end{aligned}
$$

we find that the path integral over $\mathbf{b}_{[p]}$ and $\mathbf{a}_{([p], \perp)}$ is $\frac{16 \pi^{4}}{k^{2}|p|^{2}}$. The other contributing factor, from equal and opposite nonzero momenta $[p]$, is the 
Fadeev-Popov determinant $\frac{d^{2} \mathbf{a}_{([p], \|)}}{d^{2} \mathbf{c}_{[p]}}$. The gauge transformation of $\mathbf{a}_{([p], \|)}$ is

$$
\delta \mathbf{a}_{([p], \|)}=\mathrm{i}|p| \cdot \delta \mathbf{c}_{[p]}, \quad \delta \mathbf{a}_{([p], \|)}^{*}=-\mathrm{i}|p| \cdot \delta \mathbf{c}_{[p]}^{*},
$$

so the Jacobian of the gauge transformation is given by

$$
\frac{d^{2} \mathbf{a}_{([p], \|)}}{d^{2} \mathbf{c}_{[p]}}=|p|^{2}
$$

Thus for each equal and opposite pair $[p]$ of nonzero momenta there is a cancellation of $|p|$-dependence between the dynamical Gaussian path integral and the Fadeev-Popov determinant, leaving a factor of $+\frac{16 \pi^{4}}{k^{2}}$ for each $[p]$.

Formally, then, the path integral over nonzero modes, modulo the volume of the group of gauge transformations at nonzero momentum, is

$$
\begin{aligned}
Z_{\text {nonzero }} & =\exp \left\{F_{\text {nonzero }}\right\}, \quad F_{\text {nonzero }}=\sum_{[p]} F_{[p]}, \\
F_{[p]} & =\ln \left(\frac{16 \pi^{4}}{k^{2}}\right)=-\ln \left(\frac{k^{2}}{16 \pi^{4}}\right)
\end{aligned}
$$

The set of equal and opposite pairs $[p]$ in the sum above is indexed by a set of half the momenta. Since the summand is invariant under $p \rightarrow-p$, it is easier to halve the summand and let the sum run over all momenta:

$$
F_{\text {nonzero }}=\sum_{p} F_{p}, \quad k F_{p} \equiv \frac{1}{2} F_{[p]}=+\ln \left(\frac{4 \pi^{2}}{k}\right)=-\ln \left(\frac{k}{4 \pi^{2}}\right) .
$$

Formally, then, the path integral over the nonzero modes $B$ and $A$, dividing out by the volume of gauge group at nonzero momenta, is given by

$$
\begin{aligned}
Z_{\text {nonzero }} & =\exp \left\{-\mathcal{F} \cdot \ln \left(\frac{k}{4 \pi^{2}}\right)\right\}, \\
\mathcal{F} & \equiv \sum_{p \neq 0} 1 .
\end{aligned}
$$

The quantity $\mathcal{F}$ awaits regularization and renormalization. For the moment we assert that $\mathcal{F}=-1+q \cdot \mathrm{vol}_{2}$ for any local renormalization procedure, 
where $\operatorname{vol}_{2}$ is the volume $4 \pi^{2} r_{1} r_{2}=2 \pi \beta r_{1}$ of the two-torus, and $q$ is a counterterm adjusting the effective vacuum energy density:

$$
q=\rho_{\Lambda} / \ln \left(\frac{k}{4 \pi^{2}}\right) .
$$

The choice of $q$ (or equivalently $\rho_{\Lambda}$ ) is a local counterterm and the magnitude of its finite piece is inherently ambiguous in the absence of some symmetry principle to determine it. Since the classical action is scale invariant, we are motivated to choose the value of $q$ that restores scale-invariance, namely $q=0$. The magnitude of the non-extensive piece of $\mathcal{F}$ cannot be absorbed into a local counterterm, is unambiguously determined, and should be the same for any local renormalization procedure to define $\mathcal{F}$. Thus we will choose a renormalization procedure of the general form

$$
\mathcal{F} \rightarrow \mathcal{F}_{\text {regulated }}+(\Delta q) \cdot \operatorname{vol}_{2}
$$

where $(\Delta q)$ is always chosen so that the extensive piece of $\mathcal{F}$ for whatever regulator we choose:

$$
\Delta q=-\lim _{\operatorname{vol}_{2} \rightarrow \infty} \frac{\mathcal{F}_{\text {regulated }}}{\text { vol }_{2}} .
$$

Then we exploit the fact that the summand is just a constant 1 , and thus infrared-finite for small $|p|$, to write $\mathcal{F}$ as -1 plus a counterterm plus a factorized sum, where each sum depends only on the momentum in a single direction:

$$
\mathcal{F}_{\text {regulated }}=-1+(\Delta q) \operatorname{vol}_{2}+\mathcal{F}_{1, \text { regulated }} \mathcal{F}_{2, \text { regulated }}
$$

For any local regulator characterized by a scale $\Lambda$, the sums $\mathcal{F}_{1 \text {,regulated and }}$

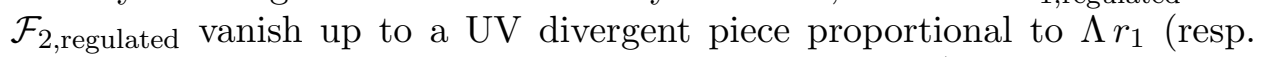
$\Lambda r_{2}$ ), as well as terms vanishing more quickly than $\Lambda^{-1}$. Thus for appro-

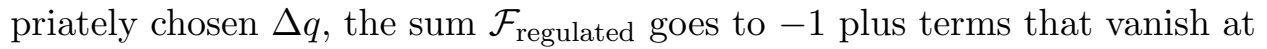
least as quickly as a negative power of $\Lambda$ when $\Lambda$ is sent to $\infty$.

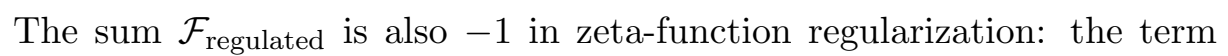
$\mathcal{F}_{1 \text {,regulated }}$ is defined by

$$
\begin{aligned}
\mathcal{F}_{1, \text { regulated }} & \equiv \sum_{p_{1}} \mu^{2 s}\left(g^{11}\left(p_{1}\right)^{2}\right)^{-s}=\left(\mu r_{1}\right)^{2 s} \sum_{n_{1}} n_{1}^{-2 s} \\
& =\left(\mu r_{1}\right)^{2 s}\left(1+2 \sum_{n_{\geq} 1} n_{1}^{-2 s}\right)=\left(\mu r_{1}\right)^{2 s}(1+2 \zeta(2 s))
\end{aligned}
$$


where $\zeta$ is the Riemann zeta function. This sum is convergent for $s>\frac{1}{2}$ but is defined uniquely by analytic continuation for all values $s \neq 1$. Removing the regulator corresponds to evaluating $s=0$. Since $\zeta(0)=-\frac{1}{2}$ we have $\mathcal{F}_{1 \text {,regulated }}=0$, and likewise $\mathcal{F}_{2 \text {,regulated }}=0$. Thus there is no need for counterterms in zeta-function regularization: $\Delta q=0$ and $\mathcal{F}=-1$.

So we have found that the appropriately renormalized value of $\mathcal{F}$ is simply -1 , and thus the renormalized path integral over nonzero modes, dividing appropriately by the nonzero mode gauge group measure, is

$$
Z_{\text {nonzero }}=\frac{k}{4 \pi^{2}} .
$$

The partition function over nonzero modes alone is nonlocal in all directions, does not have a Hilbert space interpretation and need not be integer, which is as it should be. To derive an appropriate Hilbert space interpretation of the vacuum amplitude, we need to include the contributions of the zero modes.

\section{B.4 Integral over the zero modes}

We now compute the volume of the zero modes of $A_{i}$ and $B$, and divide by the volume of the zero mode of $\chi$. The measure for the dynamical zero modes is just given by $\prod_{I=1,2} d a_{0, I} d b_{0}$ and the measure for the $\chi$ zero mode is $d c_{0}$. For both integrals the integrand is 1 and all that remains is to compute the region of integration.

The zero mode pieces of the dynamical fields $A_{i}, B$, and the gauge parameter $\chi$ are

$$
\begin{aligned}
\left.A_{i}\right|_{\text {zero mode }} & =\sum_{I} \phi_{i,(0, I)} a_{(0, I)}, \\
\left.B\right|_{\text {zero mode }} & =\phi_{0} b_{0}, \\
\left.\chi\right|_{\text {zero mode }} & =\phi_{0} c_{0},
\end{aligned}
$$

where $\phi_{0}$ and $\phi_{(0, I)}$ are zero modes of the Laplacian satisfying the orthonormality conditions. We take

$$
\phi_{0}=\frac{1}{\sqrt{\mathrm{vol}_{2}}}, \quad k \phi_{i,(0, I)}=\delta_{i I} \sqrt{\frac{g_{i i}}{\mathrm{vol}_{2}}} .
$$


To determine the fundamental region for the Wilson lines $a_{(0, I)}$, recall that the Wilson lines are identified under large gauge transformations,

$$
\left.A_{i}\right|_{\text {zero mode }} \sim A_{i}+\frac{2 \pi}{\Delta x^{i}},
$$

where $\Delta x^{i}$ is the extent of the coordinate $x^{i}$. In terms of the orthonormalized zero modes, this translates into

$$
a_{(0, I)} \sim a_{(0, I)}+\frac{2 \pi \delta_{i I} \sqrt{\mathrm{vol}_{2}}}{\sqrt{g_{i i}} \Delta x^{i}}=\frac{2 \pi \delta_{i I} \sqrt{\mathrm{vol}_{2}}}{L_{i}},
$$

where $L_{i} \equiv \sqrt{g_{i i}} \Delta x^{i}$ is the physical length of the cycle in the $x^{i}$ direction.

As for the zero modes of $B$ and $\chi$, both are identified under $2 \pi$, and their zero modes have the same normalization, so their zero modes have the same identifications:

$$
b_{0} \sim b_{0}+2 \pi \sqrt{\mathrm{vol}_{2}}, \quad k \quad c_{0} \sim c_{0}+2 \pi \sqrt{\operatorname{vol}_{2}} .
$$

So the total partition function for the zero modes is

$$
Z_{\text {zero }}=\frac{Z_{a_{0}} Z_{b_{0}}}{Z_{c_{0}}}
$$

The $b_{0}$ and $c_{0}$ zero mode integrals are the same,

$$
Z_{b_{0}}=\int_{0}^{\frac{2 \pi}{\sqrt{\text { vol }_{2}}}} d b_{0}=Z_{c_{0}}
$$

so their ratio is unity, and we are left with contributions from the $a_{0}$ zero modes:

$$
Z_{\text {zero }}=Z_{a_{0}}=\int_{0}^{\frac{2 \pi \sqrt{\mathrm{vol}_{2}}}{L_{1}}} \int_{0}^{\frac{2 \pi \sqrt{\mathrm{vol}_{2}}}{L_{2}}} d^{2} a_{(0, I)}=\frac{4 \pi^{2} \mathrm{vol}_{2}}{L_{1} L_{2}} .
$$

The product of the lengths of cycles of a rectangular torus is equal to the volume, $L_{1} L_{2}=\operatorname{vol}_{2}$, so

$$
Z_{\text {zero }}=4 \pi^{2}
$$

Thus the total partition function over the torus is the product of the zero mode and renormalized nonzero mode path integrals:

$$
Z=Z_{\text {zero }} Z_{\text {nonzero }}=\left(4 \pi^{2}\right) \frac{k}{4 \pi^{2}}=k,
$$


independent of $r_{1}$ and $r_{2}$. So we conclude that the BF theory has exactly $k$ quantum states, all of the same energy, which can be set exactly to zero by a choice of counterterm for the two-dimensional vacuum energy density. We see that the BF theory at level $k \geq 2$ is the minimal Lagrangian realization of a quantum field theory with $k$ degenerate vacua - it is minimal in the sense that it contains only the degenerate vacuua, and nothing else.

\section{B.5 Hilbert space interpretation of the vacuum amplitude}

The vacuum amplitude is purely topological: with the appropriate choice of vacuum energy density counterterm, the partition function is independent of $r_{1}$ and $r_{2}$. Interpreting the Euclidean vacuum amplitude on the torus as a thermal partition function at temperature $\beta^{-1}=\frac{1}{2 \pi r_{2}}$, we see that the dimension of the Hilbert space is $k$, and all states have exactly zero energy. Note, however, that even if we had chosen a different value for $\Delta q$, we would have had $k$ degenerate states, with common energy $E=2 \pi r_{1} \rho_{\Lambda}=$ $2 \pi r_{1}(\Delta q) / \ln \left(\frac{k}{4 \pi^{2}}\right)$.

\section{B.6 Spectrum and commutation relations of local operators and line operators}

The operators in question are

$$
\mathcal{O}_{n}(x) \equiv: \exp (\operatorname{in} B(x)):,
$$

which clearly obey $\mathcal{O}_{n} \cdot \mathcal{O}_{m}=\mathcal{O}_{n+m}$. We also have the Wilson line operators

$$
W_{n} \equiv: \exp (\operatorname{in} \oint A):,
$$

with the line integral taken over a spatial cycle. As we shall show shortly, the Wilson line operators obey simple equal-time commutation relations with the local operators $\mathcal{O}_{m}(x)$ :

$$
W_{n} \mathcal{O}_{m}=\xi^{n m} \mathcal{O}_{m} W_{n}
$$

for $\xi=\exp (-2 \pi \mathrm{i} / k)$. As a result, $W$ and $\mathcal{O}$ commute like clock and shift operators.

Given the dimension of the Hilbert space and the fact that the operators $W$ and $\mathcal{O}$ are invertible, it follows that the operators $W$ and $\mathcal{O}$ not 
only commute but actually act as clock and shift operators in the standard $k$-dimensional representation. The commutation relations define an algebra whose smallest nontrivial representation is $k$-dimensional. Since the operators $W$ and $\mathcal{O}$ are invertible, their representation on the Hilbert space cannot act as zero identically, so their representation must be nontrivial, and the standard $k$-dimensional representation is the only one that is sufficiently small.

\section{B.7 Commutation relations}

Now we shall compute the commutation relations between the Wilson line operators and the $\mathcal{O}_{n}$. We will work in timelike gauge: $A_{0}=0$. In this case, we can take the action to be

$$
S=\frac{k}{2 \pi} \int B \partial_{0} A_{1}
$$

hence the conjugate momenta are

$$
\pi_{A_{1}}=\frac{k B}{2 \pi}, \quad \pi_{B}=-\frac{k A_{1}}{2 \pi},
$$

so we have the equal-time commutators

$$
\left[B\left(x_{1}\right), A_{1}\left(x_{2}\right)\right]=-\mathrm{i} \frac{2 \pi}{k} \delta\left(x_{1}-x_{2}\right) .
$$

From this one immediately derives

$$
\left[A_{1}(x), f(B(y))\right]=\frac{2 \pi \mathrm{i}}{k} f^{\prime}(B(y)) \delta(x-y) .
$$

Define

$$
L=\oint d x A_{1}(x)
$$

so that

$$
[L, f(B)]=\frac{2 \pi \mathrm{i}}{k} f^{\prime}(B),
$$

so in particular

$$
\left[L, \mathrm{e}^{\mathrm{i} \alpha B}\right]=-\frac{2 \pi \alpha}{k} \mathrm{e}^{\mathrm{i} \alpha B}
$$


for any constant $\alpha$. It is then straightforward to compute that

$$
\mathrm{e}^{\mathrm{i} \beta L} \mathrm{e}^{\mathrm{i} \alpha B} \mathrm{e}^{-\mathrm{i} \beta L}=\exp \left(-\frac{2 \pi \mathrm{i} \alpha \beta}{k}\right) \mathrm{e}^{\mathrm{i} \alpha B}
$$

for any constants $\alpha, \beta$. Thus, in particular,

$$
W_{n} \mathcal{O}_{m}=\exp \left(-\frac{2 \pi \mathrm{i} m n}{k}\right) \mathcal{O}_{m} W_{n} .
$$

We have already seen that the dimension of the Hilbert space of states in this theory is $k$. Now we see that this $k$-dimensional Hilbert space carries a minimum-dimensional representation of the finite-dimensional analogue of the Heisenberg algebra, generated by clock and shift operators at level $k$, which are generated by natural local operators and line operators of the theory.

Concretely, the operator $\mathcal{O}_{n}$ acts on the Hilbert space as

$$
\mathcal{O}_{n} \sim\left[\begin{array}{ccccc}
1 & 0 & 0 & \cdots & 0 \\
0 & \xi^{n} & 0 & \cdots & 0 \\
0 & 0 & \xi^{2 n} & \cdots & 0 \\
\vdots & \vdots & \vdots & & \vdots \\
0 & 0 & 0 & \cdots & \xi^{(k-1) n}
\end{array}\right]
$$

Note also that the $\mathcal{O}_{n}$ exhaust the set of linearly independent local operators, as opposed to line operators, in the theory, and therefore that the state-operator correspondence holds in this theory, despite its unfamiliar features: the dimension of the Hilbert space is $k$, matching the number of independent $\mathcal{O}_{n}$.

Having established the action of the $\mathcal{O}_{n}$ on the $k$-dimensional Hilbert space, it is then possible from linear combinations of the $\mathcal{O}_{n}$ to construct $k$ independent projection operators that are also local operators, which confirms the decomposition hypothesis that we have conjectured to hold in general for two-dimensional conformal theories not satisfying cluster decomposition [14]. For example, the operator $P_{0}$ projecting onto states invariant under the continuous $B \rightarrow B+\epsilon$ symmetry is given by

$$
P_{0}=\frac{1}{k} \sum_{i=0}^{k-1} \mathcal{O}_{i} .
$$


Of course, projection operators always exist in any finite-dimensional (or even separable) Hilbert space. The existence of the projectors has special significance, indicating the failure of cluster decomposition, only because they are local operators.

\section{B.8 Direct demonstration of non-cluster-decomposition}

We can also demonstrate the failure of cluster decomposition directly in this theory, without considering Wilson lines; we can simply compute the correlation function of two local $\mathcal{O}$-operators made from $B$, and note that the correlation function is not equal to the product of one-point functions, even when the spacelike separation between the operators becomes arbitrarily large. Correlation functions containing only $B$ 's are particularly simple, receiving only contributions from the zero modes of $B$-nonzero modes do not contribute. This is because $B$ is a field that enters only linearly in the Lagrangian. In terms of Feynman diagrams, the propagator is purely anti-diagonal between $B$ and $A$ :

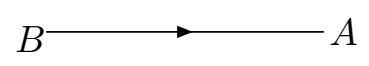

and there are no interaction vertices. Thus, the expectation value of a set of $B$ nonzero-modes is equal to its classical value, i.e., the value where all nonzero modes of $B$ are set to zero, because in the absence of $A$ modes the external $B$ lines have nowhere to terminate. This argument does not apply to zero modes of $B$ because these modes do not have a well-defined propagator and there is no diagrammatic calculation of their correlation functions.

One implication is that $\mathcal{O}_{n} \cdot \mathcal{O}_{m}=\mathcal{O}_{n+m}$. In particular, this means that

$$
\left\langle\mathcal{O}_{n}\right\rangle=\left\langle\mathcal{O}_{1}^{n}\right\rangle
$$

and from integrating over the circle of $B$ zero modes, we find that

$$
\left\langle\mathcal{O}_{1}^{n}\right\rangle=\langle 1\rangle \delta_{n, 0 \bmod k} .
$$

The result above shows directly that cluster decomposition does not hold in the $\mathrm{BF}$ theory at level $k \geq 2$. If cluster decomposition were to hold, it would mean that

$$
\lim _{x \rightarrow \infty}\left\langle\mathcal{O}_{1}(x) \mathcal{O}_{-1}(0)\right\rangle=\lim _{x \rightarrow \infty} \frac{\left\langle\mathcal{O}_{1}(x)\right\rangle\left\langle\mathcal{O}_{-1}(0)\right\rangle}{\langle 1\rangle} .
$$


On the other hand, from the results above, we know that

$$
\begin{aligned}
\left\langle\mathcal{O}_{1}(x) \mathcal{O}_{-1}(0)\right\rangle & =\langle 1\rangle, \\
\left\langle\mathcal{O}_{ \pm 1}(x)\right\rangle & =0 \quad k(\text { unless } k=1),
\end{aligned}
$$

so the property (B.42) does not hold for $k \geq 2$ : the operators $\mathcal{O}_{ \pm 1}$ are correlated with one another at arbitrary spacelike separation, as expected from a summation over multiple degenerate vacua labeled by expectation values of the $\mathcal{O}_{ \pm 1}$.

\section{References}

[1] E. Witten and J. Bagger, Quantization of Newton's constant in certain supergravity theories, Phys. Lett. B 115 (1982), 202-206.

[2] Z. Komargodski and N. Seiberg, Comments on the Fayet-Iliopoulos term in field theory and supergravity, J. High Energy Phys. 0906 (2009), 007, arXiv:0904.1159.

[3] Z. Komargodski and N. Seiberg, From linear SUSY to constrained superfields, J. High Energy Phys. 0909 (2009), 066, arXiv: 0907.2441.

[4] Z. Komargodski and N. Seiberg, Comments on supercurrent multiplets, supersymmetric field theories and supergravity, J. High Energy Phys. 1007 (2010), 017, arXiv:1002.2228.

[5] D. Butter, Conserved currents and Fayet-Iliopoulos terms in supergravity, arXiv: 1003.0249.

[6] T. Dumitrescu, Z. Komargodski and M. Sudano, Global symmetries and D-terms in supersymmetric field theories, arXiv:1007.5352.

[7] N. Seiberg, Modifying the sum over topological sectors and constraints on supergravity, arXiv:1005.0002.

[8] J. Distler and E. Sharpe, Quantization of Fayet-Iliopoulos parameters in supergravity, arXiv:1008.0419.

[9] T. Banks and N. Seiberg, Symmetries and strings in field theory and gravity, arXiv: 1011.5120.

[10] S. Katz, T. Pantev and E. Sharpe, D-branes, orbifolds, and Ext groups, Nucl. Phys. B 673 (2003), 263-300, arXiv: hep-th/0212218.

[11] T. Pantev and E. Sharpe, Notes on gauging noneffective group actions, arXiv:hep-th/0502027.

[12] T. Pantev and E. Sharpe, String compactifications on Calabi-Yau stacks, Nucl. Phys. B 733 (2006), 233-296, arXiv:hep-th/0502044. 
[13] T. Pantev and E. Sharpe, GLSM's for gerbes (and other toric stacks), Adv. Theor. Math. Phys. 10 (2006), 77-121, arXiv:hep-th/ 0502053.

[14] S. Hellerman, A. Henriques, T. Pantev, E. Sharpe and M. Ando, Cluster decomposition, T-duality, and gerby CFTs, Adv. Theor. Math. Phys. 11 (2007), 751-818, arXiv: hep-th/0606034.

[15] A. Caldararu, J. Distler, S. Hellerman, T. Pantev and E. Sharpe, Non-birational twisted derived equivalences in abelian GLSMs, Comm. Math. Phys. 294 (2010), 605-645, arXiv:0709.3855.

[16] T. Pantev and E. Sharpe, Heterotic strings on gerbes, to appear.

[17] R. Karp, On the $\mathbf{C}^{n} / \mathbf{Z}_{m}$ fractional branes, J. Math. Phys. 50 (2009), 022304, arXiv:hep-th/0602165.

[18] C. Herzog and R. Karp, On the geometry of quiver gauge theories: stacking exceptional collections, arXiv:hep-th/0605177.

[19] E. Sharpe, Derived categories and stacks in physics, contribution to the Proceedings of the ESI research Conference on Homological Mirror Symmetry (Vienna, Austria, June 2006), arXiv:hep-th/ 0608056.

[20] E. Sharpe, Landau-Ginzburg models, gerbes, and Kuznetsov's homological projective duality, to appear in the Proceedings of Topology, $\mathbf{C}^{*}$ Algebras, String Duality (Texas Christian University, May pp. 18-22, 2009).

[21] E. Sharpe, GLSM's, gerbes, and Kuznetsov's homological projective duality, contribution to the Proceedings of Quantum Theory and Symmetries 6, arXiv:1004.5388.

[22] W.-M. Chen and Y.-B. Ruan, A new cohomology theory for orbifold, Comm. Math. Phys. 248 (2004), 1-31, arXiv:math/0004129.

[23] D. Abramovich, T. Graber and A. Vistoli, Gromov-Witten theory of Deligne-Mumford stacks, Amer. J. Math. 130 (2008), 1337-1398, arXiv:math. AG/0603151.

[24] T. Coates, A. Corti, Y. Lee and H. Tseng, The quantum orbifold cohomology of weighted projective spaces, Acta. Math. 202 (2009), 139-193, arXiv: math. AG/0608481.

[25] E. Mann, Orbifold quantum cohomology of weighted projective spaces, J. Algebraic Geom. 17 (2008), 137-166, arXiv:math.AG/ 0610965.

[26] A. Vistoli, Intersection theory on algebraic stacks and on their moduli spaces, Inv. Math. 97 (1989), 613-670.

[27] T. Gomez, Algebraic stacks, Proc. Indian Acad. Sci. Math. Sci. 111 (2001), 1-31, arXiv:math.AG/9911199. 
[28] G. Laumon and L. Moret-Bailly, Champs algébriques, Springer, Berklin, 1999.

[29] K. Hori and C. Vafa, Mirror symmetry, arXiv:hep-th/0002222.

[30] D. Morrison and R. Plesser, Towards mirror symmetry as duality for two-dimensional abelian gauge theories, Nucl. Phys. Proc. Suppl. 46 (1996), 177-186, arXiv:hep-th/9508107.

[31] C. Vafa and E. Witten, On orbifolds with discrete torsion, J. Geom. Phys. 15 (1995), 189-214, arXiv:hep-th/9409188.

[32] A. Kapustin, Holomorphic reduction of $\mathcal{N}=2$ gauge theories, Wilson't Hooft operators, and S-duality, arXiv: hep-th/0612119.

[33] E. Andreini, Y. Jiang and H.-H. Tseng, On Gromov-Witten theory of root gerbes, arXiv:0812.4477.

[34] E. Andreini, Y. Jiang and H.-H. Tseng, Gromov-Witten theory of product stacks, arXiv:0905.2258.

[35] E. Andreini, Y. Jiang and H.-H. Tseng, Gromov-Witten theory of etale gerbes, $i$ : root gerbes, arXiv:0907.2087.

[36] H.-H. Tseng, On degree zero elliptic orbifold Gromov-Witten invariants, arXiv:0912.3580.

[37] A. Gholampour and H.-H. Tseng, On Donaldson-Thomas invariants of threefold stacks and gerbes, arXiv:1001.0435.

[38] X. Tang and H.-H. Tseng, Duality theorems of étale gerbes on orbifolds, arXiv: 1004.1376.

[39] A. Kapustin and E. Witten, Electric-magnetic duality and the geometric Langlands program, arXiv:hep-th/0604151.

[40] E. Witten, Mirror symmetry, Hitchin's equations, and Langlands duality, arXiv:0802.0999.

[41] R. Donagi and T. Pantev, Langlands duality for Hitchin systems, arXiv:math.AG/0604617.

[42] T. Pantev, private communication.

[43] P. Pouliot, Chiral duals of non-chiral susy gauge theories, Phys. Lett. B 359 (1995), 108-113, arXiv:hep-th/9507018.

[44] P. Pouliot and M. Strassler, A chiral $S U(N)$ gauge theory and its non-chiral Spin(8) dual, Phys. Lett. B 370 (1996), 76-82, arXiv:hep-th/9510228.

[45] M. Strassler, Duality, phases, spinors and monopoles in $S O(n)$ and Spin(n) gauge theories, J. High Energy Phys. 9809 (1998), 017, arXiv:hep-th/9709081.

[46] M. Strassler, On phases of gauge theories and the role of non-BPS solitons in field theory, arXiv:hep-th/9808073. 
[47] J. de Boer, R. Dijkgraaf, K. Hori, A. Keurentjes, J. Morgan, D. Morrison and S. Sethi, Triples, fluxes, and strings, Adv. Theor. Math. Phys. 4 (2002), 995-1186, arXiv:hep-th/0103170.

[48] M. Green, J. Schwarz and E. Witten, Superstring theory, volume I, Cambridge University Press, Cambridge, 1987.

[49] D. Gross, J. Harvey, E. Martinec and R. Rohm, Heterotic string theory I: the free heterotic string, Nucl. Phys. B 256 (1985), 253-284.

[50] P. Ginsparg, Applied conformal field theory, in Fields, strings and critical phenomena (Les Houches 1988), North-Holland, Amsterdam, 1990, pp. 1-168, arXiv:hep-th/9108028.

[51] C. Vafa, Quantum symmetries of string vacua, Mod. Phys. Lett. A 4 (1989), 1615-1626.

[52] E. Witten, D-branes and K theory, J. High Energy Phys. 9812 (1998), 019, arXiv:hep-th/9810188.

[53] J. Distler and E. Sharpe, Heterotic compactifications with principal bundles for general groups and general levels, Adv. Theor. Math. Phys. 14 (2010), 335-398, arXiv: hep-th/0701244.

[54] M. Dine, N. Seiberg and E. Witten Fayet-Iliopoulos terms in string theory, Nucl. Phys. B 289 (1987), 589-598.

[55] J. Atick, L. Dixon and A. Sen, String calculation of Fayet-Iliopoulos D-terms in arbitrary supersymmetric compactifications, Nucl. Phys. B 292 (1987), 109-149.

[56] M. Bershadsky, T. Pantev and V. Sadov, F-theory with quantized fluxes, arXiv:hep-th/9805056.

[57] A. Knutson, private communication.

[58] C. Vafa, Geometric origin of Montonen-Olive duality, arXiv:hep-th/9707131.

[59] B. Greene, A. Shapere, C. Vafa and S.-T. Yau, Stringy cosmic strings and noncompact Calabi-Yau manifolds, Nucl. Phys. B 337 (1990), $1-36$.

[60] A. Kresch, On the geometry of Deligne-Mumford stacks, in Algebraic geometry (Seattle, 2005), Proc. Symp. Pure Math. 80, part 1, Amer. Math. Soc., Providence, Rhode Island, 2009, pp. 259-271 also available at http://www.math.uzh.ch/fileadmin/user/kresch/ publikation/geodm.pdf.

[61] A. Vilenkin and E.P.S. Shellard, Cosmic strings and other topological defects, Cambridge University Press, 1994.

[62] M. Shifman and A. Yung, Supersymmetric solitons, Cambridge University Press, 2009. 
[63] D. Mumford, J. Fogarty and F. Kirwan, Geometric invariant theory, 3rd edn, Springer-Verlag, Berlin, 1994.

[64] P.E. Newstead, Introduction to moduli problems and orbit spaces, Tata Institute of Fundamental Research, Springer-Verlag, Berlin, 1978.

[65] F. Kirwan, Cohomology of quotients in symplectic and algebraic geometry, Princeton University Press, Princeton, NJ, 1984.

[66] A. Căldăraru, Derived categories of twisted sheaves on Calabi-Yau manifolds, Cornell University Ph.D. thesis, 2000. Available at http:// www.math.upenn.edu/ andreic

[67] A. Căldăraru, Derived categories of twisted sheaves on elliptic threefolds, arXiv:math/0012083.

[68] A. Căldăraru, Non-fine moduli spaces of sheaves on K3 surfaces, arXiv:math/0108180.

[69] A. Căldăraru, S. Katz and E. Sharpe, D-branes, $B$ fields, and Ext groups, Adv. Theor. Math. Phys. 7 (2004), 381-404, arXiv: hep-th/0302099.

[70] J. Distler, Notes on $\mathcal{N}=2$ sigma models, in String theory and quantum gravity 1992 (Trieste, 1992), pp. 234-256, arXiv: hep-th/9212062.

[71] G. Moore and P. Nelson, Anomalies in nonlinear sigma models, Phys. Rev. Lett. 53 (1984), 1519-1522.

[72] G. Moore and P. Nelson, The etiology of sigma model anomalies, Comm. Math. Phys. 100 (1985), 83-132.

[73] A. Manohar, G. Moore and P. Nelson, A comment on sigma model anomalies, Phys. Lett. B 152 (1985), 68-74.

[74] J. Brodie, P. Cho and K. Intriligator, Misleading anomaly matchings?, Phys. Lett. B 429 (1998), 319-326, arXiv:hep-th/9802092.

[75] K. Intriligator and N. Seiberg, Mirror symmetry in threedimensional gauge theories, Phys. Lett. B 387 (1996), 513-519, arXiv: hep-th/9607207.

[76] A. Malmendier, Donaldson invariants of $C P^{1} \times C P^{1}$ and mock theta functions, arXiv: 1008.0175.

[77] J. Polchinski, Evaluation of the one loop string path integral, Commun. Math. Phys. 104 (1986), 37-47. 
